\title{
OBSERVABLES OF MACDONALD PROCESSES
}

\author{
ALEXEI BORODIN, IVAN CORWIN, VADIM GORIN, AND SHAMIL SHAKIROV \\ To the memory of A. Zelevinsky
}

Abstract. We present a framework for computing averages of various observables of Macdonald processes. This leads to new contour-integral formulas for averages of a large class of multilevel observables, as well as Fredholm determinants for averages of two different single level observables.

\section{Contents}

1. Introduction

2. General definitions

3. Formal Macdonald processes and observables

4. Ascending Macdonald processes

5. Appendix: E. Rains' proof of Proposition 4.10

6. Appendix: On a formal version of Theorem 4.5

Acknowledgements

\section{INTRODUCTION}

The last decade saw great success surrounding the applications of Schur processes [O1], OR] to probability (cf. [BG]). Starting with the 2011 work of [BC] (see also F1, FR ), more general Macdonald processes have proved useful in solving a number of problems in probability, including: computing exact Fredholm determinant formulas and associated asymptotics for one-point marginal distributions of the O'Connell-Yor semi-discrete directed polymer [BC], BCF] (see also [CCY], [OC], $\log$-gamma discrete directed polymer [BC], BCR] (see also [COSZ], Se]), Kardar-Parisi-Zhang / stochastic heat equation [BCF] (see also ACQ, [SS]), qTASEP [BC], BCS], BC2 and $q$-PushASEP [BP, $[\mathrm{CP}$; showing Gaussian free field fluctuations for the general $\beta$ Jacobi corners process [BG2] and constructing a multilevel extension of the general $\beta$ Dyson Brownian Motion GS.

These probabilistic systems and formulas describing them arise under various choices and limits of parameters (sometimes called degenerations) for Macdonald processes (as well as natural dynamics which behave well with respect to Macdonald processes). There are other important degenerations including the study of measures on plane partitions $[\mathrm{V}]$, random unitriangular matrices over finite fields [B], F2, GKV], Section 4], Kingman and Ewens-Pitman partition structures

\footnotetext{
Received by the editors June 10, 2013 and, in revised form, December 6, 2013 and December 16, 2013.

2010 Mathematics Subject Classification. Primary 05 E05.
} 


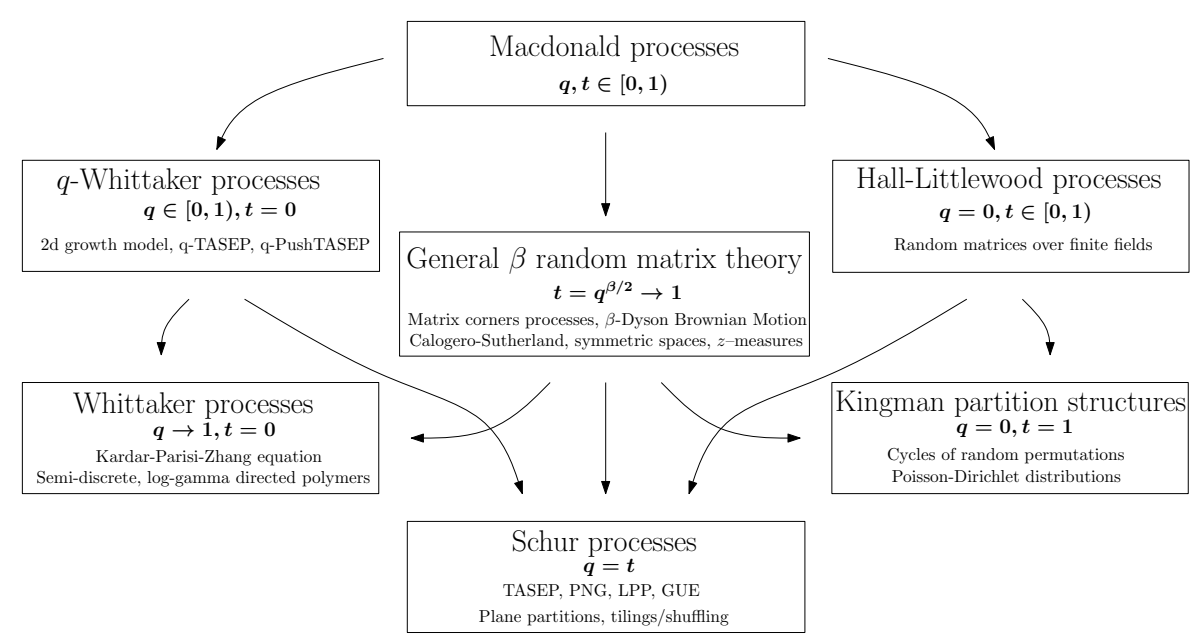

FIGURE 1. Macdonald processes unify the study of a diverse array of probabilistic systems.

[Ki], Ke1, Ke2, Chapter I], [P, $z$-measures as well as other distributions originating from the representation theory of "big" groups [BO], [OO], KOO]. Many more examples are known for the degeneration related to the Schur processes, e.g. domino/lozenge tilings and shufflings, totally asymmetric simple exclusion process, polynuclear growth model, last passage percolation, longest increasing subsequences in random permutations (see the review $[\mathrm{BG}]$ ). Figure1 indicates how these systems relate to Macdonald processes.

The integrable properties of the Macdonald symmetric functions (i.e., the family of Macdonald difference operators diagonalized by them) through which the Macdonald processes are defined naturally lead to a family of observables whose expectations can be concisely written via contour-integral formulas. This approach to studying observables of Macdonald processes was initiated in [BC], and the purpose of this paper is to develop this direction in its full generality. The family of observables (for whose expectation we have contour-integral formulas) is sufficiently rich so as to completely characterize the distribution of the Macdonald process. Thus, one could call this an integrable probabilistic system (cf. [BG]). We expect that these new results will prove useful for many of the degenerations of Macdonald processes indicated in Figure 1.

This should be compared to the fact that Schur processes of [OR (degenerations of Macdonald processes when $q=t$; see Figure 1) are known to be determinantal, meaning that all of their correlation functions are given by determinants made of a single correlation kernel. Marginal distributions of determinantal point processes are known to be expressible in terms of Fredholm determinants. Macdonald processes do not appear to be determinantal and the family of (non-local) observables which we study at the Macdonald processes level is different from those related to correlation functions (and do not degenerate to those when $q=t$ ) 1 Nevertheless we introduce two single-level observables of Macdonald processes whose expectations

\footnotetext{
${ }^{1}$ It is possible at $q=t$ to use the Macdonald process observables to recover the Schur process correlation kernel; cf. [BC, Remark 2.2.15] and [A].
} 
are given by Fredholm determinants. The first relies upon an operator of [NS] (see also [FHHSY], which is diagonalized by the Macdonald symmetric polynomials, while the second (more elementary) result relies upon the Macdonald difference operators.

Besides providing a unified framework through which to study (and discover) a variety of probabilistic systems, Macdonald processes exist at a sufficiently high algebraic level so that they may be treated as formal algebraic objects. This formal perspective, which is introduced in Section 2 and applied in detail in Section 3 , enables us to deal with a more general case of Macdonald processes than just the ascending Macdonald processes, which was the primary interest of [BC]. Already in BCFV this formal perspective has proved useful in justifying identities for which a direct analytic proof is unjustifiable due to divergences. In Section 4 we do specialize to ascending Macdonald processes and find new formulas for expectations of multilevel observables, some of which have already been applied in work on $q-$ TASEP [BCS] and general $\beta$ random matrix theory [BG].

We briefly introduce Macdonald processes and then highlight two of the results which we prove in subsequent sections. The notation and exact definitions related to symmetric functions and formal power series are introduced and explained in Section 2. As given in Definition 3.2, the formal Macdonald process $\mathbb{M P}_{N, \mathbf{A}, \mathbf{B}}^{\mathbf{f}}$ is a formal probability measure (of total weight 1 ) on $\mathbb{Y}^{N}$ (here $\mathbb{Y}$ is the set of all partitions) such that

$$
\begin{aligned}
& \mathbb{M P}_{N, \mathbf{A}, \mathbf{B}}^{\mathbf{f}}\left(\lambda^{1}, \ldots, \lambda^{N}\right) \\
& \quad=\frac{P_{\lambda^{1}}\left(A^{1}\right) \Psi_{\lambda^{2}, \lambda^{1}}\left(A^{2} ; B^{1}\right) \Psi_{\lambda^{3}, \lambda^{2}}\left(A^{3} ; B^{2}\right) \cdots \Psi_{\lambda^{N}, \lambda^{N-1}}\left(A^{N} ; B^{N-1}\right) Q_{\lambda^{N}}\left(B^{N}\right)}{\prod_{1 \leq \alpha \leq \beta \leq N} \Pi\left(A^{\alpha} ; B^{\beta}\right)},
\end{aligned}
$$

where

$$
\Psi_{\lambda, \mu}(A ; B)=\sum_{\nu \in \mathbb{Y}} P_{\lambda / \nu}(A) Q_{\mu / \nu}(B)
$$

and the factors $\Pi\left(A^{\alpha} ; B^{\beta}\right)$ are defined via

$$
\Pi(X ; Y)=\sum_{\lambda \in \mathbb{Y}} P_{\lambda}(X) Q_{\lambda}(Y) .
$$

Here $P_{\bullet}$ and $Q_{\bullet}$ are (skew) Macdonald symmetric functions (cf. Definition 2.1), and they depend on two auxiliary (Macdonald) parameters traditionally denoted by $q, t$. Though in the definition of these symmetric functions $q, t$ can be taken as formal variables, we will assume henceforth that $q, t \in[0,1)$, as this is necessary for some of the more analytic statements later in this paper.

This is called a formal probability measure since it does not assign a non-negative real probability to a given choice of $\lambda^{1}, \ldots, \lambda^{N}$, but rather assigns a formal power series in the symmetric functions of the $2 N$ sets of variables $\mathbf{A}=\left(A^{1}, \ldots, A^{N}\right)$, $\mathbf{B}=\left(B^{1}, \ldots, B^{N}\right)$ (each of these sets of variables is, itself, an infinite collection of indeterminates, so that e.g. $\left.A^{1}=\left(a_{1}^{1}, a_{2}^{1}, \ldots\right)\right)$. Alternatively, this can be thought of as formal power series in the Newton power sums $p_{k}\left(A^{i}\right)$ and $p_{k}\left(B^{j}\right)$, where $p_{k}(X)=\sum_{i}\left(x_{i}\right)^{k}$.

Define the observable $\mathcal{E}_{r}: \mathbb{Y} \rightarrow \mathbb{C}$ as (cf. Definition 3.7)

$$
\mathcal{E}_{r}(\lambda)=\lim _{N \rightarrow \infty} e_{r}\left(q^{-\lambda_{1}}, q^{-\lambda_{2}} t, \ldots, q^{-\lambda_{N}} t^{N-1}\right), \quad r \geq 1,
$$

where $e_{r}$ is the $r^{t h}$ elementary symmetric polynomial, and $\mathcal{E}_{0}(\lambda)=1$. 
For sets of indeterminates $X=\left(x_{1}, x_{2}, \ldots\right)$ and $Y=\left(y_{1}, y_{2}, \ldots\right)$, define also

$$
\text { (1.1) } \mathrm{H}(X ; Y)=\prod_{i, j} \frac{1-t x_{i} y_{j}}{1-x_{i} y_{j}} \quad \text { and } \quad \mathrm{W}(X ; Y)=\prod_{i, j} \frac{\left(1-t x_{i} y_{j}\right)\left(1-q x_{i} y_{j}\right)}{\left(1-x_{i} y_{j}\right)\left(1-q t x_{i} y_{j}\right)}
$$

These can be viewed as formal power series via $(1-u)^{-1}=\sum_{j \geq 0} u^{j}$.

The statement of Theorem 1.1 below (Theorem 3.10 in the main text) should be understood formally as an identity of symmetric power series.

Theorem 1.1. Take $N \geq 1$ and $r_{1}, \ldots, r_{N} \geq 0$. For $1 \leq m \leq N$, set $V^{m}=$ $\left\{v_{1}^{m}, \ldots, v_{r_{m}}^{m}\right\}$ and define

$$
D V^{m}=\frac{1}{\left(r_{m}\right) !(2 \pi \mathbf{i})^{r_{m}}} \operatorname{det}\left[\frac{1}{v_{i}^{m}-t v_{j}^{m}}\right]_{i, j=1}^{r_{m}} \prod_{i=1}^{r_{m}} d v_{i}^{m} .
$$

We have

$$
\begin{aligned}
& \sum_{\lambda^{1}, \ldots, \lambda^{N} \in \mathbb{Y}} \mathcal{E}_{r_{1}}\left(\lambda^{1}\right) \cdots \mathcal{E}_{r_{N}}\left(\lambda^{N}\right) \mathbb{M P}_{N, \mathbf{A}, \mathbf{B}}^{\mathbf{f}}\left(\lambda^{1}, \ldots, \lambda^{N}\right) \\
= & \oint \cdots \oint \prod_{m=1}^{N}\left(D V^{m}\right) \prod_{1 \leq \alpha \leq \beta \leq N} \mathrm{H}\left(\left(q V^{\alpha}\right)^{-1} ; B^{\beta}\right) \mathrm{H}\left(A^{\alpha} ; V^{\beta}\right) \mathrm{W}\left(\left(q V^{\alpha}\right)^{-1} ; V^{\beta}\right) .
\end{aligned}
$$

Here for a set of variables $V=\left\{v_{1}, \ldots, v_{r}\right\},(q V)^{-1}$ means the set $\left\{\left(q v_{1}\right)^{-1}, \ldots\right.$, $\left.\left(q v_{r}\right)^{-1}\right\}$. The contours of integration are a collection of positively oriented circles $\gamma_{1}, \ldots, \gamma_{m}$ of radii $R_{1}, \ldots, R_{m}$ around the origin such that $v_{i}^{\alpha}$ is integrated over $\gamma_{\alpha}$, and the radii are such that $R_{\beta}<q R_{\alpha}$ for $1 \leq \alpha<\beta \leq N$.

In what follows we call a homomorphism of an algebra into $\mathbb{C}$ a specialization. One example of a specialization of the algebra of symmetric functions in variables $x_{1}, x_{2}, \ldots$, is obtained by substituting complex numbers (subject to certain convergence conditions) in place of $x_{i}, i=1,2, \ldots$

Applying appropriate specializations to the identity of Theorem 1.1 we get an analytic statement. However, the only proof of the analytic identity we know of proceeds through the formal setting (when restricted to ascending Macdonald processes as discussed below, a direct analytic proof is known).

The ascending Macdonald process $\mathbb{M P}_{N ;\left\{a_{i}\right\} ; \rho}^{\mathbf{a}}$ (cf. Definition 4.1) is the result of specializing the $2 N$ sets of variables $\mathbf{A}=\left(A^{1}, \ldots, A^{N}\right), \mathbf{B}=\left(B^{1}, \ldots, B^{N}\right)$ in a certain way. This is now a (possibly complex-valued) measure on sequences of interlacing partitions $\lambda^{1}, \ldots, \lambda^{N}$ so that $\lambda^{i}, 1 \leq i \leq N$, has at most $i$ non-zero parts, and $\lambda^{i} \succ \lambda^{i-1}$ which means that

$$
\lambda_{1}^{i} \geq \lambda_{2}^{i-1} \geq \lambda_{2}^{i} \geq \cdots \geq \lambda_{i-1}^{i-1} \geq \lambda_{i}^{i}
$$

for $2 \leq i \leq N$. If the specializations are assumed to have certain positivity properties, then the ascending Macdonald process becomes a bona fide probability measure; cf. [BC, Section 2.2]. In Section 4.1 we provide contour-integral formulas for expectations of observables of the ascending Macdonald process of the form (in Theorem 4.5] and Theorem 4.6, respectively)

$$
\prod_{i=1}^{m} e_{r_{i}}\left(q^{\lambda_{1}^{n_{i}}} t^{n_{i}-1}, q^{\lambda_{2}^{n_{i}}} t^{n_{i}-2}, \ldots, q^{\lambda_{n_{i}}^{n_{i}}}\right) \quad \text { and } \quad \prod_{i=1}^{m} e_{r_{i}}\left(q^{-\lambda_{1}^{n_{i}}} t^{1-n_{i}}, \ldots, q^{-\lambda_{n_{i}}^{n_{i}}}\right)
$$


where $N \geq n_{1} \geq n_{2} \geq \cdots \geq n_{m} \geq 1$ and $r_{1}, \ldots, r_{m}$ are such that $0 \leq r_{i} \leq n_{i}$ for $i=1, \ldots, m$.

The projection of the ascending Macdonald process to $\lambda^{N}$ is the Macdonald measure $\mathbb{M M}_{N ;\left\{a_{i}\right\} ; \rho}^{\mathbf{a}}$, which is a complex-valued measure on $\mathbb{Y}$ which sums (over $\lambda \in \mathbb{Y}$ ) to 1 such that (replacing $\lambda^{N}$ by $\lambda$ )

$$
\mathbb{M M}_{N ;\left\{a_{i}\right\} ; \rho}^{\mathbf{a}}(\lambda)=\frac{P_{\lambda}\left(a_{1}, \ldots, a_{N}\right) Q_{\lambda}(\rho)}{\Pi\left(a_{1}, \ldots, a_{N} ; \rho\right)}
$$

Here $a_{1}, \ldots, a_{N}$ are complex numbers and $Q_{\lambda}(\rho)$ is the specialization of $Q_{\lambda}$ (cf. Section 2.3 . Given some assumptions on the $\left\{a_{i}\right\}$ and $\rho$, the normalizing term

$$
\Pi\left(a_{1}, \ldots, a_{N} ; \rho\right):=\sum_{\lambda \in \mathbb{Y}} P_{\lambda}\left(a_{1}, \ldots, a_{N}\right) Q_{\lambda}(\rho)
$$

is finite and the measure is well-defined.

The $t=0$ degeneration of Theorem 1.2 below (Theorem 4.8 in the main text) was previously discovered in [BC, Corollary 3.2.10 and Theorem 3.2.11] and served as the basis for computing exact Fredholm determinant formulas and associated asymptotics for one-point marginal distributions of the O'Connell-Yor semidiscrete directed polymer, Kardar-Parisi-Zhang / stochastic heat equation and $q$-TASEP. The proof in $[\mathrm{BC}]$ relied on the first Macdonald difference operator and its powers. Our present result uses a different operator diagonalized by the Macdonald polynomials.

Theorem 1.2. Fix $N$ non-zero complex numbers $a_{1}, \ldots, a_{N}$ and a specialization $\rho$. Then, under certain assumptions (cf. Theorem 4.8) on these parameters, as well as the contour $\gamma$, we have that the following equality holds as an identity of power series in $u$ :

$$
\sum_{\lambda \in \mathbb{Y}} \prod_{i=1}^{N} \frac{\left(q^{\lambda_{i}} t^{N-i+1} u ; q\right)_{\infty}}{\left(q^{\lambda_{i}} t^{N-i} u ; q\right)_{\infty}} \mathbb{M M}_{N,\left\{a_{i}\right\}, \rho}^{\mathbf{a}}(\lambda)=\operatorname{det}\left(I+K_{u, N,\left\{a_{i}\right\}, \rho}\right)_{L^{2}(\gamma)},
$$

where the Fredholm determinant is defined in (4.10) and

$$
\begin{aligned}
K_{u, N,\left\{a_{i}\right\}, \rho}\left(w, w^{\prime}\right) & =\sum_{v=1}^{\infty} \frac{u^{v}}{q^{v} w-w^{\prime}} \frac{\mathrm{G}_{N,\left\{a_{i}\right\}, \rho}(w)}{\mathrm{G}_{N,\left\{a_{i}\right\}, \rho}\left(q^{v} w\right)}, \\
\mathrm{G}_{N,\left\{a_{i}\right\}, \rho}(w) & =\frac{1}{\Pi(w ; \rho)} \prod_{j=1}^{N} \frac{\left(t w / a_{j} ; q\right)_{\infty}}{\left(w / a_{j} ; q\right)_{\infty}} .
\end{aligned}
$$

For the degeneration related to directed polymers (i.e., Whittaker processes; see Figure 1), this (somewhat surprisingly) converges as $N \rightarrow \infty$ to the GUE Tracy-Widom distribution $[\mathrm{BC},[\mathrm{BCF}, \overline{\mathrm{BCR}}]$. We look forward to investigating the asymptotics of the above Fredholm determinant in other degenerations indicated in Figure 1.

\section{General Definitions}

2.1. Symmetric functions. A partition $\lambda$ is a weakly decreasing sequence of nonnegative integers $\lambda_{1} \geq \lambda_{2} \geq \cdots \geq 0$, such that $\sum_{i} \lambda_{i}<\infty$. The last sum is called 
the size of a partition and is denoted $|\lambda|$. Let $\mathbb{Y}_{n}$ denote the set of all partitions of size $n$ and set

$$
\mathbb{Y}=\bigcup_{n=0}^{\infty} \mathbb{Y}_{n}
$$

where we assume $\mathbb{Y}_{0}$ to be a singleton consisting of $\varnothing$. The number of non-zero coordinates (parts) in $\lambda$ is called the length of $\lambda$ and denoted $\ell(\lambda)$.

In what follows we denote by capital letters $X, Y, A, B$ sets of variables (i.e. alphabets $)$ and by lowercase letters $x, y, \ldots$, single variables. Let $\Lambda_{X}$ denote the $\mathbb{Z}_{\geq 0}$ graded algebra (over $\mathbb{C}$ ) of symmetric functions in variables $X=\left(x_{1}, x_{2}, \ldots\right)$, which can be viewed as the algebra of symmetric polynomials in infinitely many variables $x_{1}, x_{2}, \ldots$ of bounded degree, see e.g. [M, Chapter 1] for general information on $\Lambda$. One way to view $\Lambda$ is as an algebra of polynomials in Newton power sums

$$
p_{k}(X)=\sum_{i}\left(x_{i}\right)^{k}, \quad k=1,2, \ldots
$$

For any partition $\lambda$ we set

$$
p_{\lambda}(X)=\prod_{i=1}^{\ell(\lambda)} p_{\lambda_{i}}(X) .
$$

Elements $p_{\lambda}(X), \lambda \in \mathbb{Y}$ form a linear basis in $\Lambda_{X}$.

An alternative set of algebraically independent generators of $\Lambda_{X}$ is given by the elementary symmetric functions

$$
e_{k}(X)=\sum_{i_{1}<i_{2}<\cdots<i_{k}} x_{i_{1}} x_{i_{2}} \cdots x_{i_{k}}, \quad k=1,2, \ldots
$$

We usually write $\Lambda_{X}, \Lambda_{Y}$, etc., for (isomorphic) algebras of symmetric functions in variables $X=\left(x_{1}, x_{2}, \ldots\right), Y=\left(y_{1}, y_{2}, \ldots\right)$ and so on. When the set of variables is irrelevant, we omit it from the notation and write simply $\Lambda$.

For a symmetric function $f$ let $\phi_{0}(f)$ be its free (constant, degree 0$)$ term. Clearly $\phi: \Lambda \rightarrow \mathbb{C}$ is an algebra homomorphism, and $\phi_{0}\left(p_{k}\right)=0, k=1,2, \ldots$

In what follows we fix two parameters $q, t$ and assume that they are real numbers satisfying $0<q, t<1$. Alternatively, in many places below we could have instead assumed that $q$ and $t$ are formal variables, replacing $\mathbb{C}$ in the definition of $\Lambda$ and all the following definitions with the algebra $\mathbb{C}(q, t)$ of rational function in $q$ and $t$. Since $q$ and $t$ never change throughout the paper, we omit the dependence on them from the notation.

The Macdonald scalar product $\langle\cdot, \cdot\rangle$ on $\Lambda$ is defined via

$$
\left\langle p_{\lambda}, p_{\mu}\right\rangle=\delta_{\lambda, \mu}\left(\prod_{i=1}^{\ell(\lambda)} \frac{1-q^{\lambda_{i}}}{1-t^{\lambda_{i}}}\right)\left(\prod_{i=1}^{\lambda_{1}} i^{m_{i}(\lambda)} m_{i}(\lambda) !\right),
$$

where $m_{i}(\lambda)$ is the number of parts in $\lambda$ equal to $i$.

The following definition can be found in [M, Chapter VI].

Definition 2.1. Macdonald symmetric functions $P_{\lambda}, \lambda \in \mathbb{Y}$, are a unique linear basis in $\Lambda$ such that

(1) $\left\langle P_{\lambda}, P_{\mu}\right\rangle=0$ unless $\lambda=\mu$.

(2) The leading (with respect to the reverse lexicographic order; i.e. $x_{1}^{n}$ is the largest monomial of degree $n$ ) monomial in $P_{\lambda}$ is $\prod_{i=1}^{\ell(\lambda)} x_{i}^{\lambda_{i}}$. 
Remark 1. The Macdonald symmetric function $P_{\lambda}$ is a homogeneous symmetric function of degree $|\lambda|$.

Remark 2. If we set $x_{N+1}=x_{N+2}=\cdots=0$ in $P_{\lambda}(X)$, then we arrive at symmetric polynomials $P_{\lambda}\left(x_{1}, \ldots, x_{N}\right)$ in $N$ variables, which are called the Macdonald polynomials.

Macdonald symmetric functions $Q_{\lambda}, \lambda \in \mathbb{Y}$, are dual to $P_{\lambda}$ with respect to the Macdonald scalar product:

$$
Q_{\lambda}=\left\langle P_{\lambda}, P_{\lambda}\right\rangle^{-1} P_{\lambda}, \quad\left\langle P_{\lambda}, Q_{\lambda}\right\rangle=\delta_{\lambda, \mu}, \quad \lambda, \mu \in \mathbb{Y}
$$

We also need skew Macdonald symmetric functions (see [M], Chapter VI] for details). Take two sets of variables $X=\left(x_{1}, x_{2}, \ldots\right)$ and $Y=\left(y_{1}, y_{2}, \ldots\right)$ and a symmetric function $f \in \Lambda$. Let $(X, Y)$ be the union of sets of variables $X$ and $Y$. Then we can view $f(X, Y) \in \Lambda_{(X, Y)}$ as a symmetric function in $x_{i}$ and also a symmetric function in $y_{i}$; more precisely, $f(X, Y)$ is a sum of products of symmetric functions of $x_{i}$ and symmetric functions of $y_{i}$. More formally, this operation defines a comultiplication $\Delta: \Lambda \rightarrow \Lambda \otimes \Lambda$, which turns $\Lambda$ into a bi-algebra (see e.g. [Z]).

Skew Macdonald symmetric functions $P_{\lambda / \mu}, Q_{\lambda / \mu}$ are defined as the coefficients in the expansions

$$
P_{\lambda}(X, Y)=\sum_{\mu} P_{\lambda}(X) P_{\lambda / \mu}(Y) \quad \text { and } \quad Q_{\lambda}(X, Y)=\sum_{\mu} Q_{\lambda}(X) Q_{\lambda / \mu}(Y) .
$$

Both $P_{\lambda / \mu}$ and $Q_{\lambda / \mu}$ are homogeneous symmetric functions of degree $|\lambda|-|\mu|$; moreover $P_{\lambda / \mu}=Q_{\lambda / \mu}=0$ unless $\mu \subset \lambda$ (which means that $\mu_{i} \leq \lambda_{i}$ for $i=1,2, \ldots$ ).

Remark. We do not employ the standard notation for operations on alphabets (used by many practitioners in algebraic combinatorics) in this work. We do this for two reasons. The first is that Macdonald [M] does not use this notation, and since we rely on certain formulas from his work, we have maintained his notation. The second is that we attempt to keep our formulas as explicit as possible since they may be of interest outside combinatorics to a probabilistic / mathematical physics audience. That said, let us here briefly review how some of the formulas we record below can be elegantly rewritten in terms of alphabets. We appreciate these observations, which were provided to us by the referee.

For two alphabets $X$ and $Y, X+Y$ represents their union, $X Y$ their Cartesian product, and $X /(1-q)$ is the product of $X$ and the infinite alphabet $\left\{1, q, q^{2}, \cdots\right\}$. By using this notation we can reduce the number of functions we define later. Let

$$
H(X):=\prod_{x \in X} \frac{1-t x}{1-x} .
$$

Then the notation $\Pi(X ; Y), H(X ; Y)$ and $W(X ; Y)$ introduced in (1.1) and later in (3.1) can be written as

$$
\begin{gathered}
\Pi(X ; Y)=H(X Y /(1-q)), \quad H(X ; Y)=H(X Y), \\
W(X ; Y)=H(X Y) / H(q X Y) .
\end{gathered}
$$


2.2. Topology. Given a $\mathbb{Z}_{\geq 0^{-}}$-graded algebra $A$, its topological completion $\bar{A}$ is defined as the algebra of all formal series

$$
a=\sum_{k=0}^{\infty} a_{k}, \quad a_{k} \in A, \operatorname{deg}\left(a_{k}\right)=k .
$$

For any element $a \in \bar{A}$, its lower degree $\operatorname{ldeg}(a)$ is defined as a maximal $K$ such that $a_{k}=0$ in (2.3) for all $k<K$. We equip $\bar{A}$ with a graded topology in which a sequence $b^{n}$ converges to $b \in \bar{A}$ if and only if

$$
\lim _{n \rightarrow \infty} \operatorname{ldeg}\left(b-b_{n}\right)=+\infty .
$$

In this topology $A$ is a dense subalgebra of $\bar{A}$. A completed graded algebra is defined as a topological completion of some $\mathbb{Z}_{\geq 0}$-graded algebra.

Given two graded algebras $A$ and $B$, we equip their tensor product $A \otimes B$ with a unique grading such that

$$
\operatorname{deg}(a \otimes b)=\operatorname{deg}(a)+\operatorname{deg}(b)
$$

for any homogeneous $a \in A, b \in B$. Given two completed graded algebras $\bar{A}$ and $\bar{B}$, their tensor product is defined as

$$
\bar{A} \otimes \bar{B}=\overline{A \otimes B} .
$$

Given a completed graded algebra $\bar{A}$ and a graded algebra $B$, their tensor product is defined as

$$
\bar{A} \otimes B=\overline{A \otimes_{1} B},
$$

where $A \otimes_{1} B$ is the tensor product $A \otimes B$ equipped with a unique grading such that

$$
\operatorname{deg}(a \otimes b)=\operatorname{deg}(a),
$$

for any homogeneous $a \in A, b \in B . B \otimes \bar{A}$ is defined similarly (and is canonically isomorphic to $\bar{A} \otimes B)$.

Note the difference between $\overline{A \otimes B}$ and $\overline{A \otimes_{1} B}$. For instance, if $A=\mathbb{C}[x]$ and $B=\mathbb{C}[y]$ with grading by degree of the polynomials, then $(1-x y)^{-1}=\sum_{n=0}^{\infty} x^{n} y^{n}$ belongs both to $\overline{A \otimes B}$ and $\overline{A \otimes_{1} B}$. Moreover, $(1-x)^{-1}=\sum_{n=0}^{\infty} x^{n}$ also belongs to both tensor products. However, $(1-y)^{-1}=\sum_{n=0}^{\infty} y^{n}$ belongs only to $\overline{A \otimes B}$, but not to $\overline{A \otimes_{1} B}$.

Now take three algebras $\mathcal{A}, \mathcal{B}, \mathcal{C}$ such that $\mathcal{C} \simeq \Lambda$, while $\mathcal{A}$ and $\mathcal{B}$ are either graded or completed graded algebras.

Definition 2.2. The Macdonald pairing $\langle\cdot, \cdot\rangle_{\mathcal{C}}$ is a unique (continuous) bilinear map

$$
(\mathcal{A} \otimes \mathcal{C}) \times(\mathcal{C} \otimes \mathcal{B}) \rightarrow \mathcal{A} \otimes \mathcal{B}
$$

such that

$$
\left\langle a \otimes c_{1}, c_{2} \otimes b\right\rangle_{\mathcal{C}}=\left\langle c_{1}, c_{2}\right\rangle a \otimes b .
$$

Remark. When $\mathcal{C}$ is the algebra $\Lambda_{X}$ of symmetric functions in variables $X=$ $\left(x_{1}, x_{2}, \ldots\right)$, we will also use the notation $\langle\cdot, \cdot\rangle_{X}$ for $\langle\cdot, \cdot\rangle_{\mathcal{C}}$. 
Note that our definitions imply an alternative definition of skew Macdonald symmetric functions:

$$
P_{\lambda / \mu}(X)=\left\langle P_{\lambda}(X, Y), Q_{\mu}(Y)\right\rangle_{Y} .
$$

The following property of the Macdonald pairing is crucial for us.

Proposition 2.3. Let $p_{k}$ be the Newton power sums in $\Lambda$ and let $a_{k}, b_{k}$ be two sequences of elements of graded algebras $A$ and $B$ with $\lim _{k \rightarrow \infty} \operatorname{ldeg}\left(a_{k}\right)=\lim _{k \rightarrow \infty} \operatorname{ldeg}\left(b_{k}\right)$ $=\infty$, so that

$$
\sum_{k=1}^{\infty} \frac{a_{k} p_{k}}{k} \in \bar{A} \otimes \Lambda, \quad \sum_{k=1}^{\infty} \frac{b_{k} p_{k}}{k} \in \bar{B} \otimes \Lambda
$$

Then

$$
\left\langle\exp \left(\sum_{k=1}^{\infty} \frac{a_{k} p_{k}}{k}\right), \exp \left(\sum_{k=1}^{\infty} \frac{p_{k} b_{k}}{k}\right)\right\rangle_{\Lambda}=\exp \left(\sum_{k=1}^{\infty}\left(\frac{1-q^{k}}{1-t^{k}} \cdot \frac{a_{k} b_{k}}{k}\right)\right),
$$

where the right-hand side is an element of $\overline{A \otimes B}$.

Remark. The condition (2.4) is satisfied, in particular, if $a_{k}, b_{k}$ are two sequences of homogeneous elements of graded algebras $A$ and $B$, respectively, such that $\operatorname{deg}\left(a_{k}\right)=\operatorname{deg}\left(b_{k}\right)=k$.

Proof of Proposition 2.3. Take three copies $\Lambda_{X}, \Lambda_{Y}, \Lambda_{Z}$ of the algebra of symmetric functions. Definitions imply that

$$
\left\langle\sum_{\lambda \in \mathbb{Y}} P_{\lambda}(X) Q_{\lambda}(Y), \sum_{\lambda \in \mathbb{Y}} P_{\lambda}(Y) Q_{\lambda}(Z)\right\rangle_{\Lambda_{Y}}=\sum_{\lambda \in \mathbb{Y}} P_{\lambda}(X) Q_{\lambda}(Z) .
$$

The Cauchy-type identity for Macdonald symmetric functions (see [M], Chapter VI, (2.7)]) yields

$$
\sum_{\lambda \in \mathbb{Y}} P_{\lambda}(X) Q_{\lambda}(Y)=\exp \left(\sum_{k=1}^{\infty}\left(\frac{1-t^{k}}{1-q^{k}} \cdot \frac{p_{k}(X) p_{k}(Y)}{k}\right)\right)
$$

and similarly for the sets of variables $Y$ and $Z$. Then (2.5) implies that

$$
\begin{aligned}
& \left\langle\exp \left(\sum_{k=1}^{\infty}\left(\frac{1-t^{k}}{1-q^{k}} \cdot \frac{p_{k}(X) p_{k}(Y)}{k}\right)\right), \exp \left(\sum_{k=1}^{\infty}\left(\frac{1-t^{k}}{1-q^{k}} \cdot \frac{p_{k}(Y) p_{k}(Z)}{k}\right)\right)\right\rangle_{\Lambda_{Y}} \\
& =\exp \left(\sum_{k=1}^{\infty}\left(\frac{1-t^{k}}{1-q^{k}} \cdot \frac{p_{k}(X) p_{k}(Z)}{k}\right)\right) .
\end{aligned}
$$

Now let $\varphi_{X, A}$ be a (continuous, algebra-) homomorphism from $\overline{\Lambda_{X}}$ to $\bar{A}$ such that

$$
\varphi_{X, A}: \overline{\Lambda_{X}} \rightarrow \bar{A}, \quad \varphi_{X, A}\left(p_{k}(X)\right)=\frac{1-q^{k}}{1-t^{k}} a_{k} .
$$

Also let $\varphi_{Z, B}$ be a (continuous, algebra-) homomorphism from $\overline{\Lambda_{Z}}$ to $\bar{B}$ such that

$$
\varphi_{Z, B}: \overline{\Lambda_{Z}} \rightarrow \bar{B}, \quad \varphi_{Z, B}\left(p_{k}(Z)\right)=\frac{1-q^{k}}{1-t^{k}} b_{k} .
$$

Applying $\varphi_{X, A}$ and $\varphi_{Z, B}$ to the identity (2.7) we are done. 
2.3. Formal measures. Let $\mathcal{N}$ be a countable set and let $\mathcal{A}$ be a completed graded algebra.

Definition 2.4. A formal probability measure $P$ on $\mathcal{N}$ taking values in $\mathcal{A}$ is a map $P: \mathcal{N} \rightarrow \mathcal{A}$ such that

$$
\sum_{\eta \in \mathcal{N}} P(\eta)=1
$$

The following procedure constructs a conventional probability measure on $\mathcal{N}$ from a formal one. Take a graded algebra $A$. A specialization $\rho$ is an (algebra-) homomorphism $\rho: A \rightarrow \mathbb{C}$.

An arbitrary element $g$ of $\bar{A}$ can be uniquely represented as

$$
\sum_{k=0}^{\infty} g_{k}, \quad \operatorname{deg}\left(g_{i}\right)=i, \quad i \in \mathbb{Z}_{\geq 0} .
$$

Define the $\rho$-seminorm on (a subset of) $\bar{A}$ through

$$
\|g\|_{\rho}=\sum_{k=0}^{\infty}\left|\rho\left(g_{k}\right)\right| .
$$

Let $A_{\rho} \subset \bar{A}$ denote the subset of elements with finite $\rho$-seminorm in $\bar{A}$. Clearly, $A_{\rho}$ is a subalgebra of $A$ and $\rho$ is uniquely extended to a continuous (in $\rho$-seminorm) homomorphism from $A_{\rho}$ to $\mathbb{C}$, that we denote by the same letter $\rho$.

Definition 2.5. Let $P$ be a formal probability measure on $\mathcal{N}$ taking values in $\bar{A}$. A specialization $\rho$ of $A$ is called $P$-positive if for any $\eta \in \mathcal{N}, P(\eta) \in A_{\rho}, \rho(P(\eta)) \geq 0$, and also the series $\sum_{\eta \in \mathcal{N}} P(\eta)$ converges (to 1 ) in $\rho$-seminorm.

Clearly, any $P$-positive specialization $\rho$ defines a probability measure on $\mathcal{N}$ through the formula

$$
\operatorname{Prob}(\eta)=\rho(P(\eta))
$$

\section{Formal Macdonald processes and observables}

3.1. Formal Macdonald process. For two (finite or countable) sets of variables $X=\left(x_{1}, x_{2}, \ldots\right)$ and $Y=\left(y_{1}, y_{2}, \ldots\right)$, define $\Pi(X ; Y)$ through

$$
\Pi(X ; Y)=\prod_{i, j} \frac{\left(t x_{i} y_{j} ; q\right)_{\infty}}{\left(x_{i} y_{j} ; q\right)_{\infty}},
$$

where we used the $q$-Pochhammer symbol notation:

$$
(a ; q)_{\infty}=(1-a)(1-a q)\left(1-a q^{2}\right) \cdots .
$$

If the sets $X$ and $Y$ are countable, then $\Pi(X ; Y)$ is an element of $\overline{\Lambda_{X}} \otimes \overline{\Lambda_{Y}}$; one easily checks that it is related to the generators $p_{k}(X), p_{k}(Y)$ through the following formula:

$$
\Pi(X ; Y)=\exp \left(\sum_{k=1}^{\infty} \frac{1-t^{k}}{1-q^{k}} \cdot \frac{p_{k}(X) p_{k}(Y)}{k}\right) .
$$

Note that $\Pi(X ; Y)$ can be inverted, and $\Pi(X ; Y)^{-1}$ is also an element of $\overline{\Lambda_{X}} \otimes \overline{\Lambda_{Y}}$.

$\Pi(X ; Y)$ can also be related to the Macdonald symmetric functions (see $\mathrm{M}$, Chapter VI, (2.7)] and (2.6) above):

$$
\Pi(X ; Y)=\sum_{\lambda \in \mathbb{Y}} P_{\lambda}(X) Q_{\lambda}(Y)
$$


The definition also implies that for more than two sets of variables we have

$$
\Pi\left(X^{1}, \ldots X^{k} ; Y^{1}, \ldots, Y^{m}\right)=\prod_{i=1}^{k} \prod_{j=1}^{m} \Pi\left(X^{i} ; Y^{j}\right) .
$$

Definition 3.1. Take two countable sets of variables $A$ and $B$. The formal Macdonald measure $\mathbb{M M}_{A, B}^{\mathbf{f}}$ is a formal probability measure on $\mathbb{Y}$ taking values in $\overline{\Lambda_{A}} \otimes \overline{\Lambda_{B}}$ such that

$$
\mathbb{M M}_{A, B}^{\mathbf{f}}(\lambda)=\frac{P_{\lambda}(A) Q_{\lambda}(B)}{\Pi(A ; B)}, \quad \lambda \in \mathbb{Y} .
$$

Definition 3.2. Fix an integer $N>0$ and $2 N$ sets of variables $\mathbf{A}=\left(A^{1}, \ldots, A^{N}\right)$, $\mathbf{B}=\left(B^{1}, \ldots, B^{N}\right)$. The formal Macdonald process $\mathbb{M P}_{N, \mathbf{A}, \mathbf{B}}^{\mathbf{f}}$ is a formal probability measure on $\mathbb{Y}^{N}$ taking values in

$$
\overline{\Lambda_{A^{1}}} \otimes \cdots \otimes \overline{\Lambda_{A^{N}}} \otimes \overline{\Lambda_{B^{1}}} \otimes \cdots \otimes \overline{\Lambda_{B^{N}}}
$$

such that

$$
\begin{aligned}
& \mathbb{M P}_{N, \mathbf{A}, \mathbf{B}}^{\mathbf{f}}\left(\lambda^{1}, \ldots, \lambda^{N}\right) \\
& =\frac{P_{\lambda^{1}}\left(A^{1}\right) \Psi_{\lambda^{2}, \lambda^{1}}\left(A^{2} ; B^{1}\right) \Psi_{\lambda^{3}, \lambda^{2}}\left(A^{3} ; B^{2}\right) \cdots \Psi_{\lambda^{N}, \lambda^{N-1}}\left(A^{N} ; B^{N-1}\right) Q_{\lambda^{N}}\left(B^{N}\right)}{\prod_{1 \leq \alpha \leq \beta \leq N} \Pi\left(A^{\alpha} ; B^{\beta}\right)},
\end{aligned}
$$

where

$$
\Psi_{\lambda, \mu}(A ; B)=\sum_{\nu \in \mathbb{Y}} P_{\lambda / \nu}(A) Q_{\mu / \nu}(B) .
$$

Remark. Definition 3.2 is a generalization of the definition of the Schur process of OR which arises when $q=t$.

The fact that the formal Macdonald measure is a formal probability measure on $\mathbb{Y}$ is immediate from (3.2). For the formal Macdonald process this fact is a bit more involved to see, and so we provide a proof in Lemma 3.4. In what follows we will actually use an equivalent definition of the Macdonald process which we now present.

Lemma 3.3. In the settings of Definition 3.2 we have

$$
\begin{aligned}
\mathbb{M P}_{N, \mathbf{A}, \mathbf{B}}^{\mathbf{f}}\left(\lambda^{1}, \ldots, \lambda^{N}\right) & =\frac{1}{\prod_{1 \leq \alpha \leq \beta \leq N} \Pi\left(A^{i} ; B^{j}\right)} \\
& \times Q_{\lambda^{N}}\left(B^{N}\right)\left\langle P_{\lambda^{N}}\left(A^{N}, Y^{N-1}\right), Q_{\lambda^{N-1}}\left(Y^{N-1}, B^{N-1}\right)\right\rangle_{Y^{N-1}} \\
& \times\left\langle P_{\lambda^{N-1}}\left(A^{N-1}, Y^{N-2}\right), Q_{\lambda^{N-2}}\left(Y^{N-2}, B^{N-2}\right)\right\rangle_{Y^{N-2}} \\
& \times \cdots \times\left\langle P_{\lambda^{2}}\left(A^{2}, Y^{1}\right), Q_{\lambda^{1}}\left(Y^{1}, B^{1}\right)\right\rangle_{Y^{1}} P_{\lambda^{1}}\left(A^{1}\right) .
\end{aligned}
$$


Proof. This follows from the identity (we use (2.2))

$$
\begin{aligned}
& \left\langle P_{\lambda^{k}}\left(A^{k}, Y^{k-1}\right), Q_{\lambda^{k-1}}\left(Y^{k-1}, B^{k-1}\right)\right\rangle_{Y^{k-1}} \\
& =\left\langle\sum_{\nu \in \mathbb{Y}} P_{\lambda^{k} / \nu}\left(A^{k}\right) P_{\nu}\left(Y^{k-1}\right), \sum_{\nu^{\prime}} Q_{\lambda^{k-1} / \nu^{\prime}}\left(B^{k-1}\right) Q_{\nu^{\prime}}\left(Y^{k-1}\right)\right\rangle_{Y^{k-1}} \\
& =\sum_{\nu \in \mathbb{Y}} P_{\lambda^{k} / \nu}\left(A^{k}\right) Q_{\lambda^{k-1} / \nu}\left(B^{k-1}\right)=\Psi_{\lambda^{k}, \lambda^{k-1}}\left(A^{k} ; B^{k-1}\right) .
\end{aligned}
$$

Lemma 3.4. We have

$$
\sum_{\lambda^{1}, \ldots, \lambda^{N} \in \mathbb{Y}} \mathbb{M P}_{N, \mathbf{A}, \mathbf{B}}^{\mathbf{f}}\left(\lambda^{1}, \ldots, \lambda^{N}\right)=1 .
$$

Proof. Summing (3.5) over $\lambda^{1}, \ldots, \lambda^{N}$ and using (3.2) we get

$$
\begin{array}{r}
\frac{1}{\prod_{\alpha \leq \beta} \Pi\left(A^{\alpha} ; B^{\beta}\right)}\left\langle\Pi\left(B^{N} ; A^{N}, Y^{N-1}\right),\left\langle\Pi\left(Y^{N-1}, B^{N-1} ; A^{N-1}, Y^{N-2}\right),\right.\right. \\
\left.\ldots\left\langle\Pi\left(B^{2}, Y^{2} ; A^{2}, Y^{1}\right), \Pi\left(Y^{1}, B^{1} ; A^{1}\right)\right\rangle_{Y^{1}} \ldots\right\rangle_{Y^{N-1}}
\end{array}
$$

It remains to use Proposition 2.3 in the form

$$
\left\langle\Pi\left(U ; Y^{k}\right), \Pi\left(Y^{k} ; V\right)\right\rangle_{Y^{k}}=\Pi(U ; V)
$$

for $k=1, \ldots, N-1$ and appropriate sets of variables $U$ and $V$, as well as (3.3).

Two simple yet important properties of formal Macdonald processes are summarized in the following lemmas.

Lemma 3.5. In the notation of Definition 3.2, let $\phi_{0}^{i}$ denote the map

$$
\phi_{0}^{i}: \Lambda_{A^{i+1}} \otimes \Lambda_{B^{i}} \rightarrow \mathbb{C}, \quad \phi_{0}^{i}(f \otimes g)=\phi_{0}(f) \phi_{0}(g),
$$

where $\phi_{0}$ is the constant term map, as above. Further, let $\mathbf{A}^{(j)}, \mathbf{B}^{(j)}$ denote the sets of variables $\mathbf{A} \backslash A^{j}$ and $\mathbf{B} \backslash B^{j}$, respectively. For $1 \leq i \leq N-1$ consider the formal measure

$$
M^{i}=\phi_{0}^{i}\left(\mathbb{M P}_{N, \mathbf{A}, \mathbf{B}}^{\mathbf{f}}\left(\lambda^{1}, \ldots, \lambda^{N}\right)\right) .
$$

Then for all sequences $\left(\lambda^{1}, \ldots, \lambda^{N}\right) \in \mathbb{Y}^{N}$ in the support of $M^{i}$, we have $\lambda^{i}=\lambda^{i+1}$. Furthermore, the restriction of $M^{i}$ to $\left(\lambda^{1}, \ldots, \lambda^{i-1}, \lambda^{i+1}, \ldots, \lambda^{N}\right)$ is the formal Macdonald process $\mathbb{M P}_{N-1, \mathbf{A}^{(i+1)}, \mathbf{B}^{(i)}}^{\mathbf{f}}$.

Proof. This readily follows from the identities

$$
\phi_{0}^{i}\left(\Psi_{\lambda, \mu}\left(A^{i+1}, B^{i}\right)\right)=\delta_{\lambda, \mu} \quad \text { and } \quad \phi_{0}^{i}\left(\Pi\left(A^{i+1} ; B^{j}\right)\right)=\phi_{0}^{i}\left(\Pi\left(A^{j} ; B^{i}\right)\right)=1 .
$$

Lemma 3.6. In the notation of Definition 3.2, let $\mathbf{A}^{i \cup i+1}$ denote $N-1$ sets of variables $\left\{A_{1}, A_{2}, \ldots, A_{i-1},\left(A_{i}, A_{i+1}\right), A_{i+2}, \ldots, A_{N}\right\}$; i.e. we unite $A^{i}$ and $A^{i+1}$ into a single set. Similarly define $\mathbf{B}^{i \cup i+1}$. Then the restriction of $\operatorname{MP}_{N, \mathbf{A}, \mathbf{B}}^{\mathbf{f}}\left(\lambda^{1}, \ldots, \lambda^{N}\right)$ to $\left(\lambda^{1}, \ldots, \lambda^{i-1}, \lambda^{i+1}, \ldots, \lambda^{N}\right), 1 \leq i \leq N$, is the formal Macdonald measure $\mathbb{M P}_{N-1, \mathbf{A}^{i \cup i+1}, \mathbf{B}^{i-1 \cup i}}^{\mathbf{f}}$. 
Proof. For $1<i<N$ this follows from the following identity, which is a combination of [M, Exercise 6, Section 7, Chapter VI] and (2.2):

$$
\begin{aligned}
& \sum_{\lambda^{i} \in \mathbb{Y}} \Psi_{\lambda^{i+1}, \lambda^{i}}\left(A^{i+1} ; B^{i}\right) \Psi_{\lambda^{i}, \lambda^{i-1}}\left(A^{i} ; B^{i-1}\right) \\
& =\sum_{\lambda^{i}, \mu, \nu \in \mathbb{Y}} P_{\lambda^{i+1} / \mu}\left(A^{i+1}\right) Q_{\lambda^{i} / \mu}\left(B^{i}\right) P_{\lambda^{i} / \nu}\left(A^{i}\right) Q_{\lambda^{i-1} / \nu}\left(B^{i-1}\right) \\
& =\Pi\left(A^{i} ; B^{i}\right) \sum_{\kappa, \mu, \nu \in \mathbb{Y}} P_{\lambda^{i+1} / \mu}\left(A^{i+1}\right) P_{\mu / \kappa}\left(A^{i}\right) Q_{\nu / \kappa}\left(B^{i}\right) Q_{\lambda^{i-1} / \nu}\left(B^{i-1}\right) \\
& =\Pi\left(A^{i} ; B^{i}\right) \sum_{\kappa \in \mathbb{Y}} P_{\lambda^{i+1} / \mu}\left(A^{i+1}, A^{i}\right) Q_{\lambda^{i-1} / \kappa}\left(B^{i}, B^{i-1}\right) \\
& =\Pi\left(A^{i} ; B^{i}\right) \Psi_{\lambda^{i+1}, \lambda^{i-1}}\left(\left(A^{i+1}, A^{i}\right) ;\left(B^{i}, B^{i-1}\right)\right) .
\end{aligned}
$$

For $i=1$ and $i=N$ the argument is similar.

3.2. Single level observables. For two sets of variables $X=\left(x_{1}, x_{2}, \ldots\right)$ and $Y=\left(y_{1}, y_{2}, \ldots\right)$, define

$$
\mathrm{H}(X ; Y)=\prod_{i, j} \frac{1-t x_{i} y_{j}}{1-x_{i} y_{j}}=\exp \left(\sum_{k=1}^{\infty}\left(1-t^{k}\right) \frac{p_{k}(X) p_{k}(Y)}{k}\right) .
$$

Definition 3.7. The function $\mathcal{E}_{r}: \mathbb{Y} \rightarrow \mathbb{C}$ is defined through

$$
\mathcal{E}_{r}(\lambda)=\lim _{N \rightarrow \infty} e_{r}\left(q^{-\lambda_{1}}, q^{-\lambda_{2}} t, \ldots, q^{-\lambda_{N}} t^{N-1}\right), \quad r \geq 1
$$

where $e_{r}$ is the elementary symmetric polynomial and $\mathcal{E}_{0}(\lambda)=1$.

For example,

$$
\mathcal{E}_{1}(\lambda)=\lim _{N \rightarrow \infty} \sum_{i=1}^{N} q^{-\lambda_{i}} t^{i-1}=\sum_{i=1}^{\ell(\lambda)} q^{-\lambda_{i}} t^{i-1}+\frac{t^{\ell(\lambda)}}{1-t} .
$$

Our first result is the computation of the expectation of the observables $\mathcal{E}_{r}(\lambda)$ with respect to a formal Macdonald measure.

Proposition 3.8. For two sets of variables $X$ and $Y$ we have

$$
\begin{aligned}
& \sum_{\lambda \in \mathbb{Y}} \mathcal{E}_{r}(\lambda) \mathbb{M M}_{X, Y}^{\mathbf{f}}(\lambda) \\
& \quad=\frac{1}{(2 \pi \mathbf{i})^{r} r !} \oint_{\left|w_{1}\right|=1} \ldots \oint_{\left|w_{r}\right|=1} \operatorname{det}\left[\frac{1}{w_{k}-t w_{\ell}}\right] \prod_{j=1}^{r} \mathrm{H}\left(w_{j} ; X\right) \mathrm{H}\left(\left(q w_{j}\right)^{-1} ; Y\right) d w_{j} .
\end{aligned}
$$

Let us explain how Proposition 3.8 should be understood. Clearly, the left side of (3.8) is an element of $\overline{\Lambda_{X}} \otimes \overline{\Lambda_{Y}}$. The integrand on the right-hand side of (3.8) can be (uniquely) written as a sum

$$
\sum_{k=0}^{\infty} f_{k}\left(w_{1}, \ldots, w_{r}\right) g_{k}
$$

where $f_{k}$ is a certain function of $w_{1}, \ldots, w_{r}$ and $g_{k}$ is an element of $\Lambda_{X} \otimes \Lambda_{Y}$ of degree $k$. When we integrate (3.9) termwise (with $w_{j}$ integrated over the unit circle 
$\left|w_{j}\right|=1$ ), we are left with an element of $\overline{\Lambda_{X}} \otimes \overline{\Lambda_{Y}}$. Now Proposition 3.8 claims that this element is the same as the one in the left side of (3.8).

The integrals over $w_{j}$ can be understood analytically (as complex integrals over contours) or, equivalently, they have a purely algebraic meaning. Indeed, expand $\operatorname{det}\left(\frac{1}{w_{k}-t w_{\ell}}\right)$ in the integrand in a power series using (recall that $0<t<1$ )

$$
\frac{1}{w_{k}-t w_{\ell}}=\frac{1}{w_{k}} \cdot \frac{1}{1-t w_{\ell} / w_{k}}=\frac{1}{w_{k}} \sum_{i=0}^{\infty} \frac{t^{i}\left(w_{\ell}\right)^{i}}{\left(w_{k}\right)^{i}} .
$$

Note that multiplication of series (3.10) for various indices $k$ and $\ell$ might involve summing geometric progressions with ratio $t$. After this procedure the functions $f_{k}$ in (3.9) become power series (in $w_{i}$ and $w_{i}^{-1}$ ). The contour integral of such power series over the unit circle is $(2 \pi \mathbf{i})^{r}$ times the coefficient of $\left(w_{1} \cdots w_{r}\right)^{-1}$.

Remark 1. Both left and right sides of (3.8) are symmetric under interchanging $X$ and $Y$ (a change of integration variables is needed to see the symmetry in the right side).

Remark 2. The formula is also valid for $e_{0}=1$ if we understand the empty integral as 1 .

Remark 3. If the integral is understood analytically, then the contours of integration can be chosen along the circles $\left|w_{j}\right|=R>0, j=1, \ldots, r$. The actual value of $R$ does not matter, as we can deform all the contours together without changing the value of the integral.

Remark 4. An integral representation similar to (3.8) can be found in $\underline{\mathrm{Sh}}$, Section 9] and [FHHSY, Proposition 3.6] under the name of Heisenberg Representation of the Macdonald Difference Operators.

The proof of Proposition 3.8 relies upon the following lemma.

Lemma 3.9. Take two sets of $N$ complex numbers $X=\left\{x_{i}\right\}_{i=1}^{N}$ and $Y=\left\{y_{i}\right\}_{i=1}^{N}$ such that $\left|x_{i} y_{j}\right|<1,1 \leq i, j \leq N$. Assume that there exist $r$ closed complex contours $\gamma_{1}, \ldots, \gamma_{r}$ such that the integral

$$
\frac{1}{(2 \pi \mathbf{i})^{r} r !} \oint_{\gamma_{1}} \ldots \oint_{\gamma_{r}} \operatorname{det}\left[\frac{1}{w_{k}-t w_{\ell}}\right] \prod_{j=1}^{r} \mathrm{H}\left(w_{j} ; X\right) \mathrm{H}\left(\left(q w_{j}\right)^{-1} ; Y\right) d w_{j}
$$

is equal to the sum of the residues of the integrand at $w_{j}=\left(x_{i}\right)^{-1}$ for $j=1, \ldots, r$, $i=1, \ldots, N$. Then the integral (3.11) also equals

$$
\sum_{\lambda \in \mathbb{Y}: \ell(\lambda) \leq N} e_{r}\left(q^{-\lambda_{1}} t^{0}, \ldots, q^{-\lambda_{N}} t^{N-1}\right) \frac{P_{\lambda}(X) Q_{\lambda}(Y)}{\Pi(X ; Y)} .
$$

Proof. This fact can be found in [BC, Remark 2.2.11]. The proof is based on the application of the $r^{t h}$ Macdonald difference operator in variables $X$ (see [M] Chapter VI]) to the identity

$$
\sum_{\lambda \in \mathbb{Y}: \ell(\lambda) \leq N} P_{\lambda}(X) Q_{\lambda}(Y)=\Pi(X ; Y) .
$$

See also Section 4.1 for more details. 
Proof of Proposition 3.8. Fix three reals $0<R_{1}<R_{2}<R_{3}$ such that $t R_{3}<R_{1}$. Take $N$ complex numbers $X=\left\{x_{i}\right\}_{i=1}^{N}$ and $N$ complex numbers $Y=\left\{y_{i}\right\}_{i=1}^{N}$ such that $R_{2}<\left|x_{j}\right|^{-1}<R_{3}$ and $\left|y_{j}\right| \ll R_{1}$ for all $i$. In what follows we assume that the $x_{i}$ 's are distinct, but all the formulas are readily extended to the case of equal $x_{i}$ 's by continuity.

Consider the integral

$$
\frac{1}{(2 \pi \mathbf{i})^{r} r !} \oint \ldots \oint \operatorname{det}\left[\frac{1}{w_{k}-t w_{\ell}}\right] \prod_{j=1}^{r} \mathrm{H}\left(w_{j} ; X\right) \mathrm{H}\left(\left(q w_{j}\right)^{-1} ; Y\right) d w_{j}
$$

with each $w_{j}$ being integrated over the union of circles $\left|w_{j}\right|=R_{1}$ and $\left|w_{j}\right|=R_{3}$ with the integral over $R_{1}$ being positively orientated and over $R_{3}$ begin negatively oriented. The restrictions on the variables imply that the integral is equal to the sum of the residues at $w_{i}=\left(x_{j}\right)^{-1}$ for $i=1, \ldots, r, j=1, \ldots, N$. Thus we can apply Lemma 3.9 to see that the above integral equals

$$
\sum_{\lambda \in \mathbb{Y}^{N}} e_{r}\left(q^{-\lambda_{1}}, q^{-\lambda_{2}} t, \ldots, q^{-\lambda_{N}} t^{N-1}\right) \frac{P_{\lambda}(X) Q_{\lambda}(Y)}{\Pi(X ; Y)} .
$$

Our aim is to convert the analytic identity 3.12$)=(3.13)$ into the formal identity in completed graded algebras, which constitutes Proposition 3.8 .

Note that (3.13) has a unique expansion as a (symmetric) power series in $x_{j}$ 's and $y_{j}$ 's. Any such symmetric power series can be written as a power series in $p_{k}(X), p_{k}(Y)$. As $N \rightarrow \infty$, each coefficient of the expansion for (3.13) converges to those of the left-hand side of (3.8). Therefore, it remains to show similarly that the coefficients of the expansion in power series in $p_{k}(X), p_{k}(Y)$ of (3.12) converge to the corresponding ones on the right side of (3.8). The rest of the proof is devoted to showing this.

The first step is to replace the portion of the contour of integration in which $w_{j}$ is integrated along the circle of radius $R_{3}$ by a circle of radius $R_{4} \gg 1$. We claim that the integral does not change value under this transformation. To see this fact, recall that before the deformation, the integral was equal to the sum of the residues of the integrand at points $w_{i}=\left(x_{j}\right)^{-1}, i=1, \ldots, r, j=1, \ldots, N$. Let us also compute the integral (via residues) after the deformation of the contours and show it matches. First, we integrate over $w_{1}$, getting the residues from the $N+2 r-2$ choices of poles of the integrand at $w_{1}=\left(x_{j}\right)^{-1}, j=1, \ldots, N$, and also at $w_{1}=t w_{i}, i=2, \ldots, r$, and $w_{1}=t^{-1} w_{i}, i=2, \ldots, r$. For each choice of pole, we further integrate over $w_{2}$, picking residues in a similar manner, and so on up to $w_{r}$. From this we see that the integral is expanded into a sum of residues of the integrand in (3.12) over points of the form

$$
w_{1}=\left(x_{j_{1}}\right)^{-1} t^{p_{1}}, \quad w_{2}=\left(x_{j_{2}}\right)^{-1} t^{p_{2}}, \ldots, w_{r}=\left(x_{j_{r}}\right)^{-1} t^{p_{r}}
$$

where the summation is restricted to a certain subset (which we will determine in a moment) of $j_{1}, j_{2}, \ldots, j_{r} \in\{1, \ldots, N\}$ and $p_{1}, p_{2}, \ldots, p_{r} \in \mathbb{Z}$.

In order to determine which subset of points of the form of (3.14) should be summed over, note the following properties: If at least one of the pairs coincide, i.e., $\left(j_{m}, p_{m}\right)=\left(j_{n}, p_{n}\right)$ for $m \neq n$, then the residue is zero, since the integrand has 
no singularity at such a point. This is because the Cauchy determinant (see e.g. [K]

$$
\operatorname{det}\left[\frac{1}{w_{k}-t w_{\ell}}\right]_{k, \ell=1}^{r}=\frac{t^{r(r-1) / 2}}{(1-t)^{r} w_{1} \ldots w_{r}} \prod_{k \neq \ell} \frac{w_{k}-w_{\ell}}{w_{k}-t w_{\ell}}
$$

vanishes when some of the variables coincide. Further, all $p_{i}$ should be non-positive. Indeed, no point of the kind $\left(x_{j}\right)^{-1} t^{-k}, k>0$, is inside our contours. We may further observe that the summation of residue need only be taken over points in (3.14) which are a union of strings of the form

$$
w_{i_{1}}=\left(x_{j}\right)^{-1}, \quad w_{i_{2}}=\left(x_{j}\right)^{-1} t^{-1}, \quad \ldots, \quad w_{i_{m}}=\left(x_{j}\right)^{-1} t^{1-m}
$$

(i.e., each string has the above form, but with possibly different length $m$, possibly different $j$ and disjoint variables $\left.i_{1}, i_{2}, \ldots, i_{m}\right)$. Note that if the length of any given string (i.e., $m$ in the above formula) is at least 2 , then the residue at such point vanishes. Indeed, the pole arising from the determinant in the integrand cancels out with corresponding zero of $\mathrm{H}\left(w ; x_{j}\right)$. On the other hand, if all the strings are of length 1 , then we get the same sum as before the deformation of the contours, thus proving our claim.

The integral in (3.12) with $R_{3}$ replaced now by $R_{4}$ can be written as a sum of $2^{r}$ contour-integrals over circular contours with some variables integrated over the circle of radius $R_{1}$ and others over the circle of radius $R_{4} \gg 1$. Our aim is to analyze each term and ultimately show that as $N \rightarrow \infty$ only the term with all integrations over the $R_{1}$ circle survives. Since the integrand is symmetric in $w_{j}$, it is enough to consider the case when $\left|w_{1}\right|=\left|w_{2}\right|=\cdots=\left|w_{m}\right|=R_{4}$ and $\left|w_{m+1}\right|=\cdots=\left|w_{r}\right|=R_{1}$, i.e., the integral

$$
\begin{aligned}
& \frac{1}{(2 \pi \mathbf{i})^{r} r !} \oint_{\left|w_{m+1}\right|=R_{1}} \ldots \oint_{\left|w_{r}\right|=R_{1}} \prod_{j=m+1}^{r} \mathrm{H}\left(w_{j} ; X\right) \mathrm{H}\left(\left(q w_{j}\right)^{-1} ; Y\right) \\
& \times \oint_{\left|w_{1}\right|=R_{4}} \ldots \oint_{\left|w_{m}\right|=R_{4}} \operatorname{det}\left[\frac{1}{w_{k}-t w_{\ell}}\right]_{k, \ell=1}^{r} \prod_{j=1}^{m} \mathrm{H}\left(w_{j} ; X\right) \mathrm{H}\left(\left(q w_{j}\right)^{-1} ; Y\right) \prod_{j=1}^{r} d w_{j} .
\end{aligned}
$$

Using the Cauchy determinant formula (3.15) we write:

$$
\begin{aligned}
\operatorname{det}\left[\frac{1}{w_{k}-t w_{\ell}}\right]_{k, \ell=1}^{r} & =\frac{t^{r(r-1) / 2}}{(1-t)^{r} w_{1} \ldots w_{r}} \prod_{k \neq \ell} \frac{w_{k}-w_{\ell}}{w_{k}-t w_{\ell}} \\
& =\frac{1}{(1-t)^{r} w_{1} \ldots w_{r}} \prod_{k<\ell} \frac{1-w_{\ell} / w_{k}}{1-t^{-1} w_{\ell} / w_{k}} \prod_{k<\ell} \frac{1-w_{\ell} / w_{k}}{1-t w_{\ell} / w_{k}} .
\end{aligned}
$$

Note that $\left|w_{\ell} / w_{k}\right|$ equals either 1 or $R_{1} / R_{4} \ll 1$ on our contours for $k<\ell$. Therefore,

$$
\begin{aligned}
\operatorname{det} & {\left[\frac{1}{w_{k}-t w_{\ell}}\right]_{k, \ell=1}^{r} } \\
& =\operatorname{det}\left[\frac{1}{w_{k}-t w_{\ell}}\right]_{k, \ell=m+1}^{r} \operatorname{det}\left[\frac{1}{w_{k}-t w_{\ell}}\right]_{k, \ell=1}^{m}\left(1+O\left(\left(R_{4}\right)^{-1}\right)\right) t^{m(r-m)},
\end{aligned}
$$


where the remainder $O(\cdot)$ is uniform over integration variables $w_{j}$ on our contours. For $j=1, \ldots, m$ note that

$$
\mathrm{H}\left(w_{j} ; X\right)=\prod_{k=1}^{N} \frac{1-t w_{j} x_{k}}{1-w_{j} x_{k}}=t^{N} \prod_{k=1}^{N} \frac{1-t^{-1}\left(w_{j}\right)^{-1}\left(x_{k}\right)^{-1}}{1-\left(w_{j}\right)^{-1}\left(x_{k}\right)^{-1}}=t^{N}\left(1+O\left(\left(R_{4}\right)^{-1}\right)\right)
$$

and also

$$
H\left(\left(q w_{j}\right)^{-1} ; Y\right)=1+O\left(\left(R_{4}\right)^{-1}\right) .
$$

Thus, integrating over $w_{j}, j=1, \ldots, m$, in (3.16) and then sending $R_{4} \rightarrow \infty$ we get

$$
\begin{aligned}
C(m) \cdot \frac{t^{m(N+r-m)}}{(2 \pi \mathbf{i})^{r-m} r !} \oint_{\left|w_{m+1}\right|=R_{1}} \ldots & \oint_{\left|w_{r}\right|=R_{1}} \operatorname{det}\left[\frac{1}{w_{k}-t w_{\ell}}\right]_{k, \ell=m+1}^{r} \\
& \times \prod_{j=m+1}^{r} \mathrm{H}\left(w_{j} ; X\right) \mathrm{H}\left(\left(q w_{j}\right)^{-1} ; Y\right) d w_{j},
\end{aligned}
$$

where $C(m)$ is the constant computed via

$$
C(m)=\frac{1}{(2 \pi \mathbf{i})^{m}} \oint_{\left|w_{1}\right|=R} \ldots \oint_{\left|w_{m}\right|=R} \operatorname{det}\left[\frac{1}{w_{k}-t w_{\ell}}\right]_{k, \ell=1}^{m} d w_{1} \cdots d w_{m}
$$

(note that the exact value of $R>0$ is irrelevant in the last integral).

Further, for $j=m+1, \ldots, r$ we expand the functions $\mathrm{H}\left(w_{j} ; X\right)$ into series using

$$
\mathrm{H}\left(w_{j} ; X\right)=\exp \left(\sum_{k=1}^{\infty}\left(1-t^{k}\right) \frac{\left(w_{j}\right)^{k} p_{k}(X)}{k}\right)
$$

and the power series expansion of the exponential; similarly expand $H\left(\left(q w_{j}\right)^{-1} ; Y\right)$. We get

$$
\prod_{j=m+1}^{r} \mathrm{H}\left(w_{j} ; X\right) \mathrm{H}\left(\left(q w_{j}\right)^{-1} ; Y\right)=\sum_{n=0}^{\infty} f_{n}\left(w_{m+1}, \ldots, w_{r}\right) g_{n}
$$

where $f_{n}, n \geq 0$, is an analytic function on the torus $w_{j}=R_{1}, j=m+1, \ldots, r$, and $g_{n}, n \geq 0$, is a homogeneous symmetric polynomial in $x_{1}, \ldots, x_{N}$ and $y_{1}, \ldots, y_{N}$ of degree $n$; more precisely, $g_{n}$ is a polynomial in $p_{k}(X), p_{k}(Y)$ whose coefficients do not depend on $N$ or any choices we made. Note that the convergence of expansions of $\mathrm{H}\left(w_{j} ; X\right), \mathrm{H}\left(\left(q w_{j}\right)^{-1} ; Y\right)$ is uniform with respect to varying the $\left\{w_{j}\right\}$ on their contours, the $\left\{x_{j}\right\}$ in the annulus $R_{2}<\left|x_{j}\right|^{-1}<R_{3}$ and the $\left\{y_{j}\right\}$ in some neighborhood of zero. Therefore, the order of integration in (3.17) and summation in (3.18) can be interchanged. Hence, evaluating the integrals over $w_{m+1}, \ldots, w_{r}$ transforms (3.17) into the sum

$$
t^{m(N+r-m)} \sum_{n=0}^{\infty} \hat{f}_{n} g_{n}
$$


where $g_{n}$ are as above, while $\hat{f}_{n}$ are certain coefficients which do not depend on $N$ and are given by

$$
\begin{aligned}
\hat{f}_{n}=\frac{C(m)}{(2 \pi \mathbf{i})^{r-m} r !} \oint_{\left|w_{m+1}\right|=R_{1}} \ldots \oint_{\left|w_{r}\right|=R_{1}} \operatorname{det}\left[\frac{1}{w_{k}-t w_{\ell}}\right]_{k, \ell=m+1}^{r} \\
\quad \times f_{n}\left(w_{m+1}, \ldots, w_{r}\right) \prod_{j=m+1}^{r} d w_{j} .
\end{aligned}
$$

If now $m \geq 1$, then the coefficients $t^{m(N+r-m)} \hat{f}_{n}$ vanish as $N \rightarrow \infty$. On the other hand, for $m=0$ we arrive at the right side of (3.8).

3.3. Multilevel observable. The combination of Proposition 3.8 and Proposition 2.3 gives a way to compute the expectations of very general observables of formal Macdonald processes. For two sets of variables $U=\left(u_{1}, u_{2}, \ldots\right)$ and $V=\left(v_{1}, v_{2}, \ldots\right)$ set

$$
\mathrm{W}(U ; V)=\prod_{i, j} \frac{\left(1-t u_{i} v_{j}\right)\left(1-q u_{i} v_{j}\right)}{\left(1-u_{i} v_{j}\right)\left(1-q t u_{i} v_{j}\right)}=\exp \left(\sum_{k=1}^{\infty} \frac{\left(1-t^{k}\right)\left(1-q^{k}\right)}{k} p_{k}(U) p_{k}(V)\right) \text {. }
$$

Theorem 3.10. Take $N \geq 1$ and $r_{1}, \ldots, r_{N} \geq 0$. For $1 \leq m \leq N$, set $V^{m}=$ $\left\{v_{1}^{m}, \ldots, v_{r_{m}}^{m}\right\}$ and define

$$
D V^{m}=\frac{1}{\left(r_{m}\right) !(2 \pi \mathbf{i})^{r_{m}}} \operatorname{det}\left[\frac{1}{v_{i}^{m}-t v_{j}^{m}}\right]_{i, j=1}^{r_{m}} \prod_{i=1}^{r_{m}} d v_{i}^{m}
$$

We have

$$
\begin{aligned}
& \sum_{\lambda^{1}, \ldots, \lambda^{N} \in \mathbb{Y}} \mathcal{E}_{r_{1}}\left(\lambda^{1}\right) \cdots \mathcal{E}_{r_{N}}\left(\lambda^{N}\right) \mathbb{M P}_{N, \mathbf{A}, \mathbf{B}}^{\mathbf{f}}\left(\lambda^{1}, \ldots, \lambda^{N}\right) \\
= & \oint \cdots \oint \prod_{\alpha=1}^{N}\left(D V^{\alpha}\right) \prod_{1 \leq \alpha \leq \beta \leq N} \mathrm{H}\left(\left(q V^{\alpha}\right)^{-1} ; B^{\beta}\right) \mathrm{H}\left(A^{\alpha} ; V^{\beta}\right) \mathrm{W}\left(\left(q V^{\alpha}\right)^{-1} ; V^{\beta}\right),
\end{aligned}
$$

where $\mathcal{E}_{r}(\lambda)$ is as in Definition 3.7. Note that for a set of variables $V=\left\{v_{1}, \ldots, v_{r}\right\}$, $(q V)^{-1}$ means the set $\left\{\left(q v_{1}\right)^{-1}, \ldots,\left(q v_{r}\right)^{-1}\right\}$. The contours of integration are a collection of positively oriented circles $\gamma_{1}, \ldots, \gamma_{m}$ of radii $R_{1}, \ldots, R_{m}$ around the origin such that $v_{i}^{\alpha}$ is integrated over $\gamma_{\alpha}$, and the radii are such that $R_{\beta}<q R_{\alpha}$ for $1 \leq \alpha<\beta \leq N$.

Similarly to Proposition 3.8, (3.20) should be understood as an identity of elements of $\overline{\Lambda_{A^{1}}} \otimes \cdots \otimes \overline{\Lambda_{A^{N}}} \otimes \overline{\Lambda_{B^{1}}} \otimes \cdots \otimes \overline{\Lambda_{B^{N}}}$. Such an element in the right side of (3.20) is obtained by expanding all $\mathrm{H}\left(\left(q V^{\alpha}\right)^{-1} ; B^{\beta}\right)$ and $\mathrm{H}\left(A^{\alpha} ; V^{\beta}\right)$ into symmetric series and then evaluating the integrals term-wise. This evaluation can be done either analytically (i.e., computing complex contour-integrals) or algebraically by expanding the integrals in series in variables $v_{i}^{m}$ and $\left(v_{i}^{m}\right)^{-1}$ using:

$$
\frac{1}{v_{i}^{m}-t v_{j}^{m}}=\frac{1}{v_{i}^{m}} \cdot \frac{1}{1-t v_{i}^{m} / v_{j}^{m}}=\frac{1}{v_{i}^{m}} \sum_{k=0}^{\infty} \frac{t^{k}\left(v_{j}^{m}\right)^{k}}{\left(v_{i}^{m}\right)^{k}}
$$


and for $\alpha<\beta$,

$$
\begin{aligned}
\mathrm{W}\left(\left(q v_{i}^{\alpha}\right)^{-1} ; v_{j}^{\beta}\right) & =\frac{\left(1-t v_{j}^{\beta} /\left(q v_{i}^{\beta}\right)\right)\left(1-q v_{j}^{\beta} /\left(q v_{i}^{\alpha}\right)\right)}{\left(1-v_{j}^{\beta} /\left(q v_{i}^{\alpha}\right)\right)\left(1-q t v_{j}^{\beta} /\left(q v_{i}^{\alpha}\right)\right)} \\
& =\left(1-t \frac{v_{j}^{\beta}}{q v_{i}^{\alpha}}\right)\left(1-\frac{v_{j}^{\beta}}{v_{i}^{\alpha}}\right)\left(\sum_{k=0}^{\infty}\left(\frac{v_{j}^{\beta}}{q v_{i}^{\alpha}}\right)^{k}\right) \cdot\left(\sum_{k=0}^{\infty}\left(t \frac{v_{j}^{\beta}}{v_{i}^{\alpha}}\right)^{k}\right),
\end{aligned}
$$

and then evaluating the coefficient of $\left(\prod_{m=1}^{N} \prod_{i=1}^{r_{m}} v_{i}^{m}\right)^{-1}$.

Proof of Theorem 3.10. Using Lemma 3.3, write the left-hand side of 3.20 as

$$
\begin{gathered}
\frac{1}{\prod_{1 \leq \alpha \leq \beta \leq N} \Pi\left(A^{\alpha} ; B^{\beta}\right)} \cdot\left\langle\sum_{\lambda^{N} \in \mathbb{Y}} \mathcal{E}_{r_{N}}\left(\lambda^{N}\right) Q_{\lambda^{N}}\left(B^{N}\right) P_{\lambda^{N}}\left(A^{N}, Y^{N-1}\right)\right. \\
\left\langle\sum_{\lambda^{N-1} \in \mathbb{Y}} \mathcal{E}_{r_{N-1}}\left(\lambda^{N-1}\right) Q_{\lambda^{N-1}}\left(Y^{N-1}, B^{N-1}\right) P_{\lambda^{N-1}}\left(A^{N-1}, Y^{N-2}\right)\right. \\
\vdots \\
\left\langle\sum_{\lambda^{2} \in \mathbb{Y}} \mathcal{E}_{r_{2}}\left(\lambda^{2}\right) Q_{\lambda^{2}}\left(Y^{2}, B^{2}\right) P_{\lambda^{2}}\left(A^{2}, Y^{1}\right),\right. \\
\left.\left.\left.\sum_{\lambda^{1} \in \mathbb{Y}} \mathcal{E}_{r_{1}}\left(\lambda^{1}\right) Q_{\lambda^{1}}\left(Y^{1}, B^{1}\right) P_{\lambda^{1}}\left(A^{1}\right)\right\rangle_{Y^{1}} \ldots\right\rangle_{Y^{N-2}}\right\rangle_{Y^{N-1}} .
\end{gathered}
$$

Applying Proposition 3.8 one time for each of the summations over $\lambda^{1}, \ldots, \lambda^{N}$, we find that the above expression equals

$$
\begin{gathered}
\frac{1}{\prod_{1 \leq i \leq j \leq N} \Pi\left(A^{i} ; B^{j}\right)} \cdot\left\langle\oint D V^{N} \mathrm{H}\left(V^{N} ; A^{N}, Y^{N-1}\right) \mathrm{H}\left(\left(q V^{N}\right)^{-1} ; B^{N}\right)\right. \\
\times \Pi\left(B^{N} ; A^{N}, Y^{N-1}\right), \\
\left\langle\oint D V^{N-1} \mathrm{H}\left(V^{N-1} ; A^{N-1}, Y^{N-2}\right) \mathrm{H}\left(\left(q V^{N-1}\right)^{-1} ; B^{N-1}, Y^{N-1}\right)\right. \\
\times \Pi\left(B^{N-1}, Y^{N-1} ; A^{N-1}, Y^{N-2}\right) \\
\vdots \\
\left\langle\oint D V^{2} \mathrm{H}\left(V^{2} ; A^{2}, Y^{1}\right) \mathrm{H}\left(\left(q V^{2}\right)^{-1} ; B^{2}, Y^{2}\right) \Pi\left(B^{2}, Y^{2} ; A^{2}, Y^{1}\right)\right. \\
\left.\left.\left.\oint D V^{1} \mathrm{H}\left(V^{1} ; A^{1}\right) \mathrm{H}\left(\left(q V^{1}\right)^{-1} ; B^{1}, Y^{1}\right) \Pi\left(B^{1}, Y^{1} ; A^{1}\right)\right\rangle_{Y^{1}} \ldots\right\rangle_{Y^{N-2}}\right\rangle_{Y^{N-1}}
\end{gathered}
$$

Note that if we view the integrations as algebraic operations (as is explained after Proposition 3.8), then in (3.21) using the continuity of the Macdonald pairing and of the constant term evaluation in the topology of completed graded algebras, we can interchange the order of integration and evaluating scalar products. Then we can use Proposition 2.3 . For the variables $Y^{1}$ we get (omitting all the factors 
independent of $Y^{1}$ which do not change in the scalar product evaluation)

$$
\begin{aligned}
&\left\langle\mathrm{H}\left(V^{2} ; Y^{1}\right) \Pi\left(B^{2}, Y^{2} ; Y^{1}\right)\right.\left., \mathrm{H}\left(\left(q V^{1}\right)^{-1} ; Y^{1}\right) \Pi\left(Y^{1} ; A^{1}\right)\right\rangle_{Y^{1}} \\
&=\mathrm{H}\left(V^{2} ; A^{1}\right) \mathrm{H}\left(\left(q V^{1}\right)^{-1} ; B^{2}, Y^{2}\right) \Pi\left(B^{2}, Y^{2} ; A^{1}\right) \\
& \times \exp \left(\sum_{k=1}^{\infty} \frac{\left(1-t^{k}\right)\left(1-q^{k}\right)}{k} p_{k}\left(V^{2}\right) p_{k}\left(\left(q V^{1}\right)^{-1}\right)\right) .
\end{aligned}
$$

Note that by (3.19)

$$
\exp \left(\sum_{k=1}^{\infty} \frac{\left(1-t^{k}\right)\left(1-q^{k}\right)}{k} p_{k}\left(V^{2}\right) p_{k}\left(\left(q V^{1}\right)^{-1}\right)\right)=W\left(V^{2} ;\left(q V^{1}\right)^{-1}\right)
$$

if we assume $\left|v_{i}^{2} /\left(q v_{j}^{1}\right)\right|<1$ when expanding $W\left(V^{2} ;\left(q V^{1}\right)^{-1}\right)$ in power series. This gives the same restriction on the contours as the one in Theorem 3.10. In the next step we evaluate the scalar product for the variables $Y^{2}$ and find (again omitting factors independent of $Y^{2}$ )

$$
\begin{aligned}
\left\langle\mathrm{H}\left(V^{3} ; Y^{2}\right) \Pi\left(B^{3}, Y^{3} ; Y^{2}\right), \mathrm{H}\left(\left(q V^{2}\right)^{-1},\left(q V^{1}\right)^{-1} ; Y^{2}\right) \Pi\left(Y^{2} ; A^{1}, A^{2}\right)\right\rangle_{Y^{2}} \\
=\mathrm{H}\left(V^{3} ; A^{1}, A^{2}\right) \mathrm{H}\left(\left(q V^{2}\right)^{-1},\left(q V^{1}\right)^{-1} ; B^{3}, Y^{3}\right) \Pi\left(B^{3}, Y^{3} ; A^{1}, A^{2}\right) \\
\times \mathrm{W}\left(V^{3} ;\left(q V^{2}\right)^{-1},\left(q V^{1}\right)^{-1}\right) .
\end{aligned}
$$

Further evaluating scalar products for variables $Y^{3}, \ldots, Y^{N-1}$ we arrive at the claimed formula.

3.4. Simple corollaries. Let us give two corollaries of Theorem 3.10.

Corollary 3.11. Take any $M$ integers $1 \leq k_{1} \leq k_{2} \leq \cdots \leq k_{M} \leq N$ and $M$ positive integers $r_{1}, \ldots, r_{M}$. With the notation and contours as in Theorem 3.10 we have

$$
\begin{aligned}
\sum_{\lambda^{1}, \ldots, \lambda^{N}} \mathcal{E}_{r_{1}}\left(\lambda^{k_{1}}\right) \cdots \mathcal{E}_{r_{M}}\left(\lambda^{k_{M}}\right) \mathbb{M P}_{N, \mathbf{A}, \mathbf{B}}^{\mathbf{f}}\left(\lambda^{1}, \ldots, \lambda^{N}\right) \\
=\oint \cdots \oint \prod_{m=1}^{M}\left(D V^{m}\right) \prod_{1 \leq \alpha, \beta \leq M: k_{\alpha} \leq \beta} \mathrm{H}\left(\left(q V^{\alpha}\right)^{-1} ; B^{\beta}\right) \\
\quad \times \prod_{1 \leq \alpha, \beta \leq M: \alpha \leq k_{\beta}} \mathrm{H}\left(A^{\alpha} ; V^{\beta}\right) \prod_{1 \leq \alpha<\beta \leq M} \mathrm{~W}\left(\left(q V^{\alpha}\right)^{-1} ; V^{\beta}\right) .
\end{aligned}
$$

Remark. The difference from Theorem 3.10 is that now we compute expectations of various products and powers of $\mathcal{E}_{r}\left(\lambda^{m}\right)$; thus (3.22) is more general than (3.20).

Proof of Corollary 3.11. The proof is a combination of Theorem 3.10 with Lemma 3.5.

Take $2(N+M)$ auxiliary sets of variables $\mathbf{C}=\left(C_{1}, \ldots, C_{N+M}\right)$ and $\mathbf{D}=$ $\left(D_{1}, \ldots, D_{N+M}\right)$. Let $\lambda^{1}, \ldots, \lambda^{N+M}$ be distributed according to $\mathbb{M P}_{M, \mathbf{C}, \mathbf{D}}^{\mathbf{f}}$ and apply Theorem 3.10 to it with the sequence of numbers $r_{i}^{\prime}, i=1, \ldots, N+M$ (they were called $r_{i}$ in Theorem 3.10, but we use $r_{i}^{\prime}$ here to avoid confusion with numbers $r_{i}$ of Corollary 3.11), obtained as follows: we set the first $k_{1} r_{i}^{\prime}$ 's to equal 0 , the next one (i.e., $\left.\left.r_{k_{1}+1}^{\prime}\right)\right)$ is $r_{1}$, then we take $k_{2}-k_{1}$ zeroes, then $r_{2}, \ldots$, and so on until $r_{M}$ and finally $N-k_{M}$ zeroes. Applying to the result $\phi_{0}^{i-1}$ (as in Lemma 3.5) 
for all indices $1 \leq i \leq N+M$ such that $r_{i} \neq 0$ and renaming the remaining sets of variables $C_{j}, D_{j}$ into $A_{i}$ and $B_{i}$, we get (3.22).

For example, if $N=1, M=2$, and $k_{1}=k_{2}=1, r_{1}=r_{2}=1$, then we start from $\mathbf{C}=\left(C_{1}, C_{2}, C_{3}\right), \mathbf{D}=\left(D_{1}, D_{2}, D_{3}\right)$ and the corresponding Macdonald process. Application of Theorem 3.10 with $r^{\prime}=(0,1,1)$ gives the contour-integral formula for

$$
\sum_{\lambda^{1}, \lambda^{2}, \lambda^{3} \in Y} \mathcal{E}_{1}\left(\lambda^{2}\right) \mathcal{E}_{1}\left(\lambda^{3}\right) \mathbb{M P}_{3 ; \mathbf{C}, \mathbf{D}}^{\mathbf{f}}\left(\lambda^{1}, \lambda^{2}, \lambda^{3}\right)
$$

When we apply $\phi_{0}^{2}$ and $\phi_{0}^{1}$ to (3.23), the summation becomes restricted to $\lambda^{1}=$ $\lambda^{2}=\lambda^{3}$, and after renaming the sets of variables we arrive at the desired contourintegral formula for

$$
\sum_{\lambda^{1} \in \mathbb{Y}}\left(\mathcal{E}_{1}\left(\lambda^{1}\right)\right)^{2} \mathbb{M P}_{1 ; \mathbf{A}, \mathbf{B}}^{\mathbf{f}}\left(\lambda^{1}\right)
$$

Corollary 3.12. In the notation of Theorem 3.10 , let $c_{1}, \ldots, c_{N}$ be any numbers (or formal variables) and set

$$
d_{i}=\prod_{j=i}^{N} c_{i}, \quad d_{N+1}=1 .
$$

We have

$$
\begin{aligned}
& \sum_{\lambda^{1}, \ldots, \lambda^{N}}\left(\left(c_{1}\right)^{\left|\lambda^{1}\right|} \mathcal{E}_{r_{1}}\left(\lambda^{1}\right)\right) \cdots\left(\left(c_{N}\right)^{\left|\lambda^{N}\right|} \mathcal{E}_{r_{N}}\left(\lambda^{N}\right)\right) \operatorname{MP}_{N, \mathbf{A}, \mathbf{B}}^{\mathbf{f}}\left(\lambda^{1}, \ldots, \lambda^{N}\right) \\
& \quad=\prod_{1 \leq \alpha \leq \beta \leq N} \frac{\Pi\left(d_{\alpha} A^{\alpha} ;\left(d_{\beta+1}\right)^{-1} B^{\beta}\right)}{\Pi\left(A^{\alpha} ; B^{\beta}\right)} \oint \cdots \oint \prod_{m=1}^{N}\left(D V^{m}\right) \\
& \times \prod_{1 \leq \alpha \leq \beta \leq N} \mathrm{H}\left(\left(q V^{\alpha}\right)^{-1} ;\left(d_{\beta+1}\right)^{-1} B^{\beta}\right) \mathrm{H}\left(d_{\alpha} A^{\alpha} ; V^{\beta}\right) \mathrm{W}\left(\left(q V^{\alpha}\right)^{-1} ; V^{\beta}\right) .
\end{aligned}
$$

Proof. The homogeneity of (skew) Macdonald symmetric functions implies that

$$
\begin{array}{r}
\frac{d_{1}^{\left|\lambda^{1}\right|} d_{2}^{\left|\lambda^{2}\right|-\left|\lambda^{1}\right|} \cdots d_{N}^{\left|\lambda^{N}\right|-\left|\lambda^{N-1}\right|}}{\prod_{\alpha \leq \beta} \Pi\left(A^{\alpha} ; B^{\beta}\right)} P_{\lambda^{1}}\left(A^{1}\right) \Psi_{\lambda^{2}, \lambda^{1}}\left(A^{2} ; B^{1}\right) \\
\cdots \Psi_{\lambda^{N}, \lambda^{N-1}}\left(A^{N} ; B^{N-1}\right) Q_{\lambda^{N}}\left(B^{N}\right) \\
=\frac{1}{\prod_{\alpha \leq \beta} \Pi\left(A^{\alpha} ; B^{\beta}\right)} P_{\lambda^{1}}\left(d_{1} A^{1}\right) \Psi_{\lambda^{2}, \lambda^{1}}\left(d_{2} A^{2} ;\left(d_{2}\right)^{-1} B^{1}\right) \\
\cdots \Psi_{\lambda^{N}, \lambda^{N-1}}\left(d_{N} A^{N} ;\left(d_{N}\right)^{-1} B^{N-1}\right) Q_{\lambda^{N}}\left(B^{N}\right) .
\end{array}
$$

Thus, we can use Lemma 3.3 and (3.20) to compute the sum in the left side of (3.24) and we reach the desired result.

Of course, one can also combine Corollaries 3.11 and 3.12. We leave the resulting statement to an interested reader. 


\section{Ascending Macdonald processes}

Let us focus on a special case of Macdonald processes, which is very useful in applications; cf. [BC].

For any complex number $a$, let $\phi_{a}$ be an algebra homomorphism $\phi_{a}: \Lambda \rightarrow \mathbb{C}$ (i.e., a specialization) such that $\phi_{a}\left(p_{k}\right)=a^{k}$. In other words, $\phi_{a}$ is the substitution $x_{1}=$ $a, x_{2}=x_{3}=\cdots=0$ into a symmetric function $f(X)$ in variables $X=\left(x_{1}, x_{2}, \ldots\right)$. In particular, $\phi_{0}$ is the evaluation of the free term of a symmetric polynomial, as before. Let us also fix an arbitrary specialization $\rho: \Lambda \rightarrow \mathbb{C}$. As explained in Section 2.3. $\phi_{a}$ and $\rho$ can be naturally extended so as to act on elements of $\Lambda_{\phi_{a}} \subset \bar{\Lambda}$ and $\Lambda_{\rho} \subset \bar{\Lambda}$, respectively. In what follows (and where it leads to no confusion) we write $f(a)$ and $f(\rho)$ for $\phi_{a}(f)$ and $\rho(f)$, respectively.

Recall that values $\mathbb{M P}_{N, \mathbf{A}, \mathbf{B}}^{\mathbf{f}}\left(\lambda^{1}, \ldots, \lambda^{N}\right)$ of a formal Macdonald process belong to the completed tensor product

$$
\overline{\Lambda_{A^{1}}} \otimes \cdots \otimes \overline{\Lambda_{A^{N}}} \otimes \overline{\Lambda_{B^{1}}} \otimes \cdots \otimes \overline{\Lambda_{B^{N}}} .
$$

Definition 4.1. Take $N$ non-zero complex numbers $a_{1}, \ldots, a_{N}$ and a specialization $\rho$ such that for some $0<R<1$ we have

$$
\left|p_{k}(\rho)\right|<R^{k}, k=1,2, \ldots, \quad\left|a_{i}\right| R<1, i=1, \ldots, N .
$$

The ascending Macdonald process $\mathbb{M P}_{N ;\left\{a_{i}\right\} ; \rho}^{\mathbf{a}}$ is defined as a composition of the formal Macdonald process $\operatorname{MP}_{N, \mathbf{A}, \mathbf{B}}^{\mathbf{f}}$ and the map $\Theta$ :

$$
\Theta=\phi_{a_{1}} \otimes \phi_{a_{2}} \otimes \cdots \otimes \phi_{a_{N}} \otimes \phi_{0} \otimes \cdots \otimes \phi_{0} \otimes \rho .
$$

In other words, $\mathbb{M M}_{N ;\left\{a_{i}\right\} ; \rho}^{\mathbf{a}}$ is a complex-valued measure on $\mathbb{Y}^{N}$ which sums to 1 and such that

$$
\mathbb{M P}_{N ;\left\{a_{i}\right\} ; \rho}^{\mathbf{a}}\left(\lambda^{1}, \ldots, \lambda^{N}\right)=\frac{P_{\lambda_{1}}\left(a_{1}\right) P_{\lambda^{2} / \lambda^{1}}\left(a_{2}\right) \cdots P_{\lambda^{N} / \lambda^{N-1}}\left(a_{N}\right) Q_{\lambda^{N}}(\rho)}{\Pi\left(a_{1}, \ldots, a_{N} ; \rho\right)},
$$

where

$$
\Pi\left(a_{1}, \ldots, a_{N} ; \rho\right)=\prod_{i=1}^{N} \Pi\left(a_{i} ; \rho\right)=\prod_{i=1}^{N} \exp \left(\sum_{k=1}^{\infty} \frac{1-t^{k}}{1-q^{k}} \cdot \frac{\sum_{i=1}^{N}\left(a_{i}\right)^{k} p_{k}(\rho)}{k}\right) .
$$

Remark 1. Our restrictions on $\rho$ and $a_{i}$ ensure the absolute convergence of the series

$$
\sum_{\lambda^{1}, \ldots, \lambda^{N} \in \mathbb{Y}} P_{\lambda_{1}}\left(a_{1}\right) P_{\lambda^{2} / \lambda^{1}}\left(a_{2}\right) \cdots P_{\lambda^{N} / \lambda^{N-1}}\left(a_{N}\right) Q_{\lambda^{N}}(\rho) .
$$

Indeed, this series is a permutation of the absolutely convergent series obtained by expanding the right-hand side of (4.1) in power series in $a_{i}, p_{k}$; thus it is also absolutely convergent.

Remark 2. If $a_{i}$ are non-negative reals and $\rho$ takes non-negative values on Macdonald symmetric functions, then $\mathbb{M P}_{N ;\left\{a_{i}\right\} ; \rho}^{\mathbf{a}}$ is a probability measure, i.e., it is positive; cf. [BC, Definition 2.2.7].

Remark 3. $\mathbb{M P}_{N ;\left\{a_{i}\right\} ; \rho}^{\mathbf{a}}\left(\lambda^{1}, \ldots, \lambda^{N}\right)$ is an analytic function of the complex numbers $a_{i}, i=1, \ldots, N$.

Lemma 4.2. The support of the ascending Macdonald process $\mathbb{M P}_{N ;\left\{a_{i}\right\} ; \rho}^{\mathbf{a}}$ is (a subset of) the set of partition $\lambda^{1}, \ldots, \lambda^{N}$ such that $\ell\left(\lambda^{i}\right) \leq i$ for $i=1, \ldots, N$ and $\lambda^{i} \succ \lambda^{i-1}$ for $i=2, \ldots, N$ (thus the term "ascending"). 
Proof. This follows from the fact that for any $a \in \mathbb{C} \backslash\{0\}, P_{\lambda / \mu}(a)=0$ unless $\lambda \succ \mu$ [M], Section 7, Chapter VI].

Note that the projection of the ascending Macdonald process $\mathbb{M P}_{N ;\left\{a_{i}\right\} ; \rho}^{\mathbf{a}}$ to $\lambda^{N}$ is a Macdonald measure (cf. Lemma 3.6, [BG, Proposition 6.3], [BC, Section 2.2.2]), which we now define.

Definition 4.3. Take $N$ non-zero complex numbers $a_{1}, \ldots, a_{N}$ and a specialization $\rho$ such that for some $0<R<1$ we have

$$
\left|p_{k}(\rho)\right|<R^{k}, k=1,2, \ldots, \quad\left|a_{i}\right| R<1, i=1, \ldots, N .
$$

The Macdonald measure $\mathbb{M M}_{N ;\left\{a_{i}\right\} ; \rho}^{\mathbf{a}}$ is a complex-valued measure on $\mathbb{Y}$ which sums to 1 such that

$$
\mathbb{M M}_{N ;\left\{a_{i}\right\} ; \rho}^{\mathbf{a}}(\lambda)=\frac{P_{\lambda}\left(a_{1}, \ldots, a_{N}\right) Q_{\lambda}(\rho)}{\Pi\left(a_{1}, \ldots, a_{N} ; \rho\right)} .
$$

The projection of the ascending Macdonald process $\mathbb{M P}_{N ;\left\{a_{i}\right\} ; \rho}^{\mathbf{a}}$ to $\lambda^{1}, \ldots, \lambda^{k}$ is again an ascending Macdonald process $\mathbb{M P}_{k ;\left\{a_{i}\right\} ; \rho}^{\mathbf{a}}$; cf. Lemma 3.6 and [BC, Section $2.2 .2]$.

The rest of this section is devoted to computing expectations of observables of ascending Macdonald processes (and measures). First, we present analogues of Theorem 3.10 and Corollary 3.11 which in this case can be proved in a much simpler way that does not require the formal approach. Then we show how the expectation of another (much smaller) family of observables can be written in terms of Fredholm determinants.

4.1. Multilevel moments. Let us introduce certain difference operators which act on analytic functions in $x_{1}, \ldots, x_{N}$ invariant (symmetric) under the permutations of arguments.

For any subset $I \subset\{1, \ldots, N\}$ define

$$
A_{I}\left(x_{1}, \ldots, x_{N} ; t\right)=\prod_{i \in I} \prod_{j \notin I} \frac{x_{i}-t x_{j}}{x_{i}-x_{j}} .
$$

Define the shift operator $T_{q, i}$ through

$$
\left[T_{q, i} f\right]\left(x_{1}, \ldots, x_{N}\right)=f\left(x_{1}, \ldots, x_{i-1}, q x_{i}, x_{i+1}, \ldots, x_{N}\right) .
$$

For any $1 \leq r \leq N$ define the $r^{\text {th }}$ Macdonald difference operator $\mathfrak{M}_{N}^{r}$ through

$$
\mathfrak{M}_{N}^{r}=\sum_{I \subset\{1, \ldots, N\}:|I|=k} A_{I}\left(x_{1}, \ldots, x_{N} ; t\right) \prod_{i \in I} T_{q, i} .
$$

One of the important properties of the Macdonald difference operators is the fact that the Macdonald polynomials are their eigenfunctions; see [M], Section 4, Chapter $\mathrm{VI}]$ :

$$
\mathfrak{M}_{k}^{r} P_{\lambda}\left(x_{1}, \ldots, x_{k} ; q, t\right)=e_{r}\left(q^{\lambda_{1}} t^{k-1}, q^{\lambda_{2}} t^{k-2}, \ldots, q^{\lambda_{k}}\right) P_{\lambda}\left(x_{1}, \ldots, x_{k} ; q, t\right) .
$$

The following proposition is a key in evaluating expectations of observables of ascending Macdonald processes. 
Proposition 4.4. Fix sequences of integers $N \geq n_{1} \geq n_{2} \geq \cdots \geq n_{m} \geq 1$ and $r_{1}, \ldots, r_{m}$, such that $0 \leq r_{i} \leq n_{i}$ for $i=1, \ldots, m$. Then

$$
\begin{aligned}
\sum_{\lambda^{1}, \ldots, \lambda^{N} \in \mathbb{Y}} \prod_{i=1}^{m} e_{r_{i}}\left(q^{\lambda_{1}^{n_{i}}} t^{n_{i}-1}, q^{\lambda_{2}^{n_{i}}} t^{n_{i}-2}, \ldots, q^{\lambda_{n_{i}}^{n_{i}}}\right) \mathbb{M P}_{N ;\left\{a_{i}\right\} ; \rho}^{\mathbf{a}}\left(\lambda^{1}, \ldots, \lambda^{N}\right) \\
=\left.\frac{\mathfrak{M}_{n_{m}}^{r_{m}} \ldots \mathfrak{M}_{n_{1}}^{r_{1}} \Pi\left(x_{1}, \ldots, x_{N} ; \rho\right)}{\Pi\left(x_{1}, \ldots, x_{N} ; \rho\right)}\right|_{x_{1}=a_{1}, \ldots, x_{N}=a_{N}} .
\end{aligned}
$$

Remark. The operators $\mathfrak{M}_{n}^{r}$ do not commute for different values of $n$. Thus the order of operators in the right side of (4.3) is important.

Proof of Proposition 4.4. We may immediately replace $N$ on the right-hand side of (4.3) with $n_{1}$ since $\Pi$ is multiplicative in the $x$ variables and the difference operators act only on $x_{1}, \ldots, x_{n_{1}}$. We expand $\Pi\left(x_{1}, \ldots, x_{n_{1}} ; \rho\right)$ in a sum using a specialized version of (3.2):

$$
\Pi\left(x_{1}, \ldots, x_{n_{1}} ; \rho\right)=\sum_{\lambda \in \mathbb{Y}: \ell(\lambda) \leq n_{1}} P_{\lambda}\left(x_{1}, \ldots, x_{n_{1}}\right) Q_{\lambda}(\rho) .
$$

Apply $\prod_{i=1}^{k-1} \mathfrak{M}_{n_{i}}^{r_{i}}$ to the sum, where $k$ is a maximal number such that $n_{1}=n_{2}=$ $\cdots=n_{k-1}$. Using (4.2) we get

$$
\begin{aligned}
& \prod_{i=1}^{k-1} \mathfrak{M}_{n_{i}}^{r_{i}} \Pi\left(x_{1}, \ldots, x_{n_{1}} ; \rho\right) \\
= & \sum_{\lambda^{n_{1}} \in \mathbb{Y}: \ell(\lambda) \leq n_{1}}\left(\prod_{i=1}^{k-1} e_{r_{i}}\left(q^{\lambda_{1}^{n_{1}}} t^{n_{1}-1}, \ldots, q^{\lambda_{n_{1}}^{n_{1}}}\right)\right) P_{\lambda^{n_{1}}}\left(x_{1}, \ldots, x_{n_{1}}\right) Q_{\lambda^{n_{1}}}(\rho) .
\end{aligned}
$$

Note that since $0<q, t<1$,

$$
\left|e_{r}\left(q^{\lambda_{1}^{n_{1}}} t^{n_{1}-1}, \ldots, q^{\lambda_{n_{1}}}\right)\right| \leq\left(n_{1}\right)^{r} .
$$

Therefore, the series in (4.5) is absolutely convergent. Now substitute in (4.5) the decomposition (which is a specialized version of Definition 2.2)

$$
P_{\lambda^{n_{1}}}\left(x_{1}, \ldots, x_{n_{1}}\right)=\sum_{\lambda^{n_{k}} \in \mathbb{Y}: \ell\left(\lambda^{n_{k}}\right) \leq n_{k}} P_{\lambda^{n_{k}}}\left(x_{1}, \ldots, x_{n_{k}}\right) P_{\lambda^{n_{1}} / \lambda^{n_{k}}}\left(x_{n_{k}+1}, \ldots, x_{n_{1}}\right)
$$

and apply (again using (4.2) ) $\prod_{i=k}^{h-1} \mathfrak{M}_{n_{i}}^{r_{i}}$ to the resulting sum, where $h$ is a maximal number such that $n_{k}=n_{k+1}=\cdots=n_{h-1}$. Iterating this procedure we arrive at the desired statement.

The next two theorems express averages of a class of observables of ascending Macdonald processes through contour-integrals.

Theorem 4.5. Take $N \geq 1$, non-zero complex numbers $a_{1}, \ldots, a_{N}$, and a specialization $\rho$ such that for some $0<R<1$ we have

$$
\left|p_{k}(\rho)\right|<R^{k}, k=1,2, \ldots, \quad\left|a_{i}\right| R<1, i=1, \ldots, N .
$$

Fix sequences of integers $N \geq n_{1} \geq n_{2} \geq \cdots \geq n_{m} \geq 1$ and $r_{1}, \ldots, r_{m}$, such that $0 \leq r_{i} \leq n_{i}$ for $i=1, \ldots, m$. Suppose that there exist closed positively oriented contours $\gamma_{i}^{\alpha}, \alpha=1, \ldots, m, i=1, \ldots, r_{\alpha}$, in the complex plane such that:

(I) All contours lie inside $\mathbb{D}_{R^{-1}}=\left\{z \in \mathbb{C}|| z \mid<R^{-1}\right\}$. 
(II) All contours enclose the points $a_{1}, \ldots, a_{N}$, but not 0 .

(III) The contour $\gamma_{i}^{\alpha}$ does not enclose points $t^{-1} q^{k} a_{1}, \ldots, t^{-1} q^{k} a_{N}, k=0, \ldots$, $m-\alpha$.

(IV) The contour $\gamma_{i}^{\alpha}$ intersects neither the interior of the image of $\gamma_{j}^{\beta}$ multiplied by $q$ nor the interior of the image of $\gamma_{j}^{\beta}$ multiplied by $t^{-1}$ for $i=1, \ldots, r_{\alpha}$, $j=1, \ldots, r_{\beta}$ and $\beta>\alpha$.

Then we have

$$
\begin{aligned}
& \sum_{\lambda^{1}, \ldots, \lambda^{N}} \prod_{i=1}^{m} e_{r_{i}}\left(q^{\lambda_{1}^{n_{i}}} t^{n_{i}-1}, \ldots, q^{\lambda_{n_{i}}^{n_{i}}}\right) \mathbb{M P}_{N ;\left\{a_{i}\right\} ; \rho}^{\mathbf{a}}\left(\lambda^{1}, \ldots, \lambda^{N}\right) \\
= & \prod_{\alpha=1}^{m} \frac{1}{(2 \pi \mathbf{i})^{r_{\alpha}} r_{\alpha} !} \oint \ldots \oint \prod_{1 \leq \alpha<\beta \leq m}\left(\prod_{i=1}^{r_{\alpha}} \prod_{i=1}^{r_{\beta}} \frac{\left(t z_{i}^{\alpha}-q z_{j}^{\beta}\right)\left(z_{i}^{\alpha}-z_{j}^{\beta}\right)}{\left(z_{i}^{\alpha}-q z_{j}^{\beta}\right)\left(t z_{i}^{\alpha}-z_{j}^{\beta}\right)}\right) \\
\times & \prod_{\alpha=1}^{m}\left(\operatorname{det}\left[\frac{1}{t z_{i}^{\alpha}-z_{j}^{\beta}}\right]_{i, j=1}^{r_{\alpha}} \prod_{i=1}^{r_{\alpha}}\left(\left(\prod_{j=1}^{n_{\alpha}} \frac{t z_{i}^{\alpha}-a_{j}}{z_{i}^{\alpha}-a_{j}}\right) \frac{\Pi\left(q z_{i}^{\alpha} ; \rho\right)}{\Pi\left(z_{i}^{\alpha} ; \rho\right)} d z_{i}^{\alpha}\right)\right)
\end{aligned}
$$

where $z_{i}^{\alpha}$ is integrated over $\gamma_{i}^{\alpha}$.

Remark 1. When all $a_{i}$ are equal, $t \ll 1$ and $q$ is close to 1 , then the required contours do exist; see e.g. [BC, Figure 3.1]. However, for some specific choices of $a_{i}, t$ and $q$ the desired contours may fail to exist; see BG2 for the study of one such case.

Remark 2. In the case $n_{1}=n_{2}=\cdots=n_{m}$ this theorem is equivalent to $\mathrm{BC}$, Proposition 2.2.15].

Proof of Theorem 4.5. The proof is similar to that of [BC, Proposition 2.2.15].

We use Proposition 4.4 and sequentially apply the operators $\mathfrak{M}_{n_{i}}^{r_{i}}$. At the first step we need the following identity:

$$
\begin{aligned}
\mathfrak{M}_{n_{1}}^{r_{1}} \Pi\left(x_{1}, \ldots, x_{N} ; \rho\right) & \\
=\frac{\Pi\left(x_{1}, \ldots, x_{N} ; \rho\right)}{(2 \pi \mathbf{i})\left(r_{1}\right) !} \oint & \ldots \oint \operatorname{det}\left[\frac{1}{t z_{i}^{1}-z_{j}^{1}}\right]_{i, j=1}^{r} \\
& \times \prod_{i=1}^{r_{1}}\left(\left[\prod_{j=1}^{n_{1}} \frac{t z_{i}^{1}-x_{j}}{z_{i}^{1}-x_{j}}\right) \frac{\Pi\left(q z_{i}^{1} ; \rho\right)}{\Pi\left(z_{i}^{1} ; \rho\right)} d z_{i}^{1}\right)
\end{aligned}
$$

with $z_{i}^{1}$ integrated over $\gamma_{i}^{1}$ and with $x_{1}, \ldots, x_{N}$ being arbitrary complex numbers inside $\gamma_{i}^{1}$ such that $t^{-1} x_{i}, i=1, \ldots, N$, are outside $\gamma_{i}^{1}$ (note that we will later need $x_{i}$ to be equal to $q^{k} a_{i}$, for various $k \geq 0$, hence restriction $I I I$ on the contours). The formula (4.7) is proved by expanding the right side of (4.7) as a sum of residues; for that we need $\Pi(q z ; \rho) / \Pi(z ; \rho)$ to be analytic inside the contour, hence restriction $I$ on the contours.

We claim that the only poles of the integrand inside the contour are at the points $z_{i}^{1}=x_{j}, i=1, \ldots, r, j=1, \ldots, n$ (our choice of $\left\{x_{j}\right\}$ and restriction $I I$ on the contours guarantee that these points will always be inside, while 0 is outside). To see this observe that the only other possible singularities of the integrand arise when $z_{i_{1}}^{1}=x_{j}, z_{i_{2}}^{1}=t x_{j}, z_{i_{3}}^{1}=t^{2} x_{j}, \ldots$ or $z_{i_{1}}^{1}=x_{j}, z_{i_{2}}=t^{-1} x_{j}, z_{i_{3}}=t^{-2} x_{j}, \ldots$ (here $i_{1}, i_{2}, \ldots$ are unique elements of $\{1, \ldots, r\}$ and $j \in\left\{1, \ldots, n_{1}\right\}$ ). The first 
possibility actually does not produce a pole because of the factor $\left(t z_{i}^{1}-x_{j}\right)$ in the integrand, and the second possibility is outside the contour because of our choice of $\left\{x_{j}\right\}$. These considerations imply the claim.

The residues of the integrand at the points $z_{i}^{1}=x_{j}, i=1, \ldots, r, j=1, \ldots, n$, give the same summands as in the definition of the operator $\mathfrak{M}_{n_{1}}^{r_{1}}$ (note the factor $r_{1}$ ! which appears because we sum over subsets in the definition of $\mathfrak{M}_{n_{1}}^{r_{1}}$ and over ordered subsets when expanding the integral as a sum of residues).

Next, we apply $\mathfrak{M}_{n_{2}}^{r_{2}}$ to the right side of (4.7). Note that by linearity we can apply the difference operator under the integral. The part of the right-hand side of (4.7) which is dependent on the $\left\{x_{j}\right\}_{j=1}^{n_{2}}$ is

$\Pi\left(x_{1}, \ldots, x_{n_{2}} ; \rho\right)\left(\prod_{i=1}^{r_{1}} \prod_{j=1}^{n_{2}} \frac{t z_{i}^{1}-x_{j}}{z_{i}^{1}-x_{j}}\right)=\prod_{j=1}^{n_{2}}\left(\Pi\left(x_{j} ; \rho\right) \prod_{i=1}^{r_{1}} \frac{t z_{i}^{1}-x_{j}}{z_{i}^{1}-x_{j}}\right)=: \prod_{j=1}^{n_{2}} \Pi^{\prime}\left(x_{j}, \rho\right)$.

We can use an analogue of (4.7) to express

$$
\mathfrak{M}_{n_{2}}^{r_{2}}\left(\prod_{j=1}^{n_{2}} \Pi^{\prime}\left(x_{j} ; \rho\right)\right)
$$

using the contours $\gamma_{i}^{2}, i=1, \ldots, r_{2}$. We have

$$
\frac{\Pi^{\prime}(q z ; \rho)}{\Pi^{\prime}(z ; \rho)}=\frac{\Pi(q z ; \rho)}{\Pi(z ; \rho)} \cdot \frac{t z_{i}^{1}-q z}{z_{i}^{1}-q z} \cdot \frac{z_{i}^{1}-z}{t z_{i}^{1}-z},
$$

hence the beginning of the appearance of the product $\prod_{1 \leq \alpha<\beta \leq m}$ in (4.6). Further, $\Pi^{\prime}(q z ; \rho) / \Pi^{\prime}(z ; \rho)$ must be analytic inside the contours $\gamma_{i}^{2}$, hence the restriction $I V$ on contours.

Further iterating this procedure for $\mathfrak{M}_{n_{3}}^{r_{3}}, \ldots, \mathfrak{M}_{n_{m}}^{r_{m}}$ leads to integrals over the contours $\gamma_{i}^{3}, \ldots \gamma_{i}^{m}$ and ultimately (4.6).

The next statement is a version of Theorem 4.5 with a different set of observables.

Theorem 4.6. Take $N$ non-zero complex numbers $a_{1}, \ldots, a_{N}$ and a specialization $\rho$ such that for some $0<R<1$ and $m \geq 1$ we have

$$
\left|p_{k}(\rho)\right|<R^{k}, k=1,2, \ldots, \quad\left|a_{i}\right| R<q^{m}, i=1, \ldots, N .
$$

Fix two sequences of integers $N \geq n_{1} \geq n_{2} \geq \cdots \geq n_{m} \geq 1$ and $r_{1}, \ldots, r_{m}$ such that $0 \leq r_{i} \leq n_{i}$ for $i=1, \ldots, m$. Suppose that there exist closed positively oriented contours $\gamma_{i}^{\alpha}, \alpha=1, \ldots, m, i=1, \ldots, r_{\alpha}$, such that:

(I) All contours lie inside $\mathbb{D}_{q R^{-1}}=\left\{z \in \mathbb{C}|| z \mid<q R^{-1}\right\}$.

(II) All contours enclose the points $a_{1}, \ldots, a_{N}$, but not 0 .

(III) The contour $\gamma_{i}^{\alpha}$ does not enclose points $t q^{-k} a_{1}, \ldots, t q^{-k} a_{N}, k=0, \ldots, m-\alpha$.

(IV) The contour $\gamma_{i}^{\alpha}$ intersects neither the interior of the image of $\gamma_{j}^{\beta}$ multiplied by $q^{-1}$ nor the interior of the image of $\gamma_{j}^{\beta}$ multiplied by $t$ for $i=1, \ldots, r_{\alpha}$, $j=1, \ldots, r_{\beta}$ and $\beta>\alpha$. 
Then we have

$$
\sum_{\lambda^{1}, \ldots, \lambda^{N} \in \mathbb{Y}} \prod_{i=1}^{m} e_{r_{i}}\left(q^{-\lambda_{1}^{n_{i}}} t^{1-n_{i}}, \ldots, q^{\left.-\lambda_{n_{i}}^{n_{i}}\right)} \mathbb{M P}_{N ;\left\{a_{i}\right\} ; \rho}^{\mathbf{a}}\left(\lambda^{1}, \ldots, \lambda^{N}\right)\right.
$$

$$
\begin{aligned}
& =\prod_{\alpha=1}^{m} \frac{1}{(2 \pi \mathbf{i})^{r_{\alpha}} r_{\alpha} !} \oint \ldots \oint \prod_{1 \leq \alpha<\beta \leq m}\left(\prod_{i=1}^{r_{\alpha}} \prod_{i=1}^{r_{\beta}} \frac{\left(z_{i}^{\alpha}-t q^{-1} z_{j}^{\beta}\right)\left(z_{i}^{\alpha}-z_{j}^{\beta}\right)}{\left(z_{i}^{\alpha}-q^{-1} z_{j}^{\beta}\right)\left(z_{i}^{\alpha}-t z_{j}^{\beta}\right)}\right) \\
& \times \prod_{\alpha=1}^{m}\left(\operatorname{det}\left[\frac{1}{t^{-1} z_{i}^{\alpha}-z_{j}^{\alpha}}\right]_{i, j=1}^{r_{\alpha}} \prod_{j=1}^{r_{\alpha}}\left(\left(\prod_{i=1}^{n_{\alpha}} \frac{t^{-1} z_{j}^{\alpha}-a_{i}}{z_{j}^{\alpha}-a_{i}}\right) \frac{\Pi\left(q^{-1} z_{j}^{\alpha} ; \rho\right)}{\Pi\left(z_{j}^{\alpha} ; \rho\right)} d z_{j}^{\alpha}\right)\right),
\end{aligned}
$$

where $z_{i}^{\alpha}$ is integrated over $\gamma_{i}^{\alpha}$.

Proof. First, note that since $0<q, t<1$,

$$
\left|e_{r}\left(q^{-\lambda_{1}} t^{1-n}, \ldots, q^{-\lambda_{n}}\right)\right| \leq q^{-|\lambda|} t^{-r} n^{r} .
$$

Therefore, the sum in the left side of (4.8) is absolutely bounded by

$$
\begin{aligned}
& \text { const } \cdot \sum_{\lambda^{1}, \ldots, \lambda^{N} \in \mathbb{Y}} q^{-m\left|\lambda^{N}\right|}\left|\mathbb{M P}_{N ;\left\{a_{i}\right\} ; \rho}^{\mathbf{a}}\left(\lambda^{1}, \ldots, \lambda^{N}\right)\right| \\
& =\text { const } \cdot \sum_{\lambda^{1}, \ldots, \lambda^{N} \in \mathbb{Y}}\left|\mathbb{M P}_{N ;\left\{a_{i}\right\} ; q^{-m} \rho}^{\mathbf{a}}\left(\lambda^{1}, \ldots, \lambda^{N}\right)\right|,
\end{aligned}
$$

where $q^{-m} \rho$ is the specialization defined by

$$
p_{k}\left(q^{-m} \rho\right)=q^{-k m} p_{k}(\rho),
$$

which, by the hypothesis of the theorem (that $\left.\left|p_{k}(\rho)\right|<R^{k}\right)$ implies that $\left|p_{k}\left(q^{-m} \rho\right)\right|$ $<\left(q^{-m} R\right)^{k}$. Therefore

$$
\left|\left(a_{i}\right)^{k} p_{k}\left(q^{-m} \rho\right)\right|<r^{k}
$$

for some $r<1$. Now the absolute convergence of the series in the Definition 4.1 implies that the series in the left side of (4.8) is absolutely convergent.

The definition of Macdonald symmetric functions $P_{\lambda}$ implies that they are invariant under the change of parameters $(q, t) \leftrightarrow\left(q^{-1}, t^{-1}\right)$ (see [M, Section VI.4, (4.14),(iv)]). In other words, restoring the notational dependence of $P_{\lambda}$ on $(q, t)$, we have

$$
P_{\lambda}(\cdot ; q, t)=P_{\lambda}\left(\cdot ; q^{-1}, t^{-1}\right) .
$$

Therefore, if we replace all instances of $q$ and $t$ in the definition of the operator $\mathfrak{M}_{k}^{r}$ by $q^{-1}$ and $t^{-1}$, respectively, and denote the resulting operator through $\widehat{\mathfrak{M}}_{k}^{r}$, then

$$
\widehat{\mathfrak{M}}_{k}^{r} P_{\lambda}\left(x_{1}, \ldots, x_{k} ; q, t\right)=e_{r}\left(q^{-\lambda_{1}} t^{1-k}, \ldots, q^{-\lambda_{k}}\right) P_{\lambda}\left(x_{1}, \ldots, x_{k} ; q, t\right) .
$$

It follows that an analogue of Proposition 4.4 holds for $\widehat{\mathfrak{M}}_{k}^{r}$ and we can repeat the proof of Theorem 4.5 (as long as all the series involved converge). The final formula (4.8) is obtained from the result of Theorem 4.5) through the formal inversion of $q$ and $t$.

One interesting limit of the above formulas can be obtained by sending $t \rightarrow 0$. The limits of Macdonald symmetric functions themselves as $t \rightarrow 0$ are known as $q$-Whittaker functions; cf. [GLO]. Denote

$$
\mathbb{Q W W P}_{N ;\left\{a_{i}\right\} ; \rho}^{\mathbf{a}}=\lim _{t \rightarrow 0} \mathbb{M P}_{N ;\left\{a_{i}\right\} ; \rho}^{\mathbf{a}} .
$$


A straightforward limit of (the case $r_{i}=1, i=1, \ldots, m$, of) Theorem 4.5 gives the following statement.

Corollary 4.7. Take $N$ non-zero complex numbers $a_{1}, \ldots, a_{N}$ and a specialization $\rho$ such that for some $0<R<1$ we have

$$
\left|p_{k}(\rho)\right|<R^{k}, k=1,2, \ldots, \quad\left|a_{i}\right| R<1, i=1, \ldots, N .
$$

Fix a sequence of integers $N \geq n_{1} \geq n_{2} \geq \cdots \geq n_{1} \geq 1$. Suppose that there exist complex closed positively oriented contours $\gamma_{\alpha}, \alpha=1, \ldots, m$, such that:

(I) All contours lie inside $\mathbb{D}_{R^{-1}}=\left\{z \in \mathbb{C}|| z \mid<R^{-1}\right\}$.

(II) All contours enclose the points $a_{1}, \ldots, a_{N}$, but not 0 .

(III) $\gamma_{\alpha}$ contour does not intersect the interior of the image of $\gamma_{\beta}$ multiplied by $q$ for $\beta>\alpha$.

Then we have

$$
\begin{aligned}
& \text { (4.9) } \sum_{\lambda^{1}, \ldots, \lambda^{N} \in \mathbb{Y}} \prod_{i=1}^{m} q^{\lambda_{n_{i}}^{n_{i}}} \mathbb{Q W W P}_{N ;\left\{a_{i}\right\} ; \rho}^{\mathbf{a}}\left(\lambda^{1}, \ldots, \lambda^{N}\right) \\
& =\frac{(-1)^{m} q^{m(m-1) / 2}}{(2 \pi \mathbf{i})^{m}} \oint \ldots \oint \prod_{1 \leq \alpha<\beta \leq m} \frac{z_{\alpha}-z_{\beta}}{z_{\alpha}-q z_{\beta}} \prod_{\alpha=1}^{m}\left(\prod_{i=1}^{n_{\alpha}} \frac{a_{i}}{a_{i}-z_{\alpha}}\right) \frac{\widetilde{\Pi}\left(q z_{\alpha} ; \rho\right)}{\widetilde{\Pi}\left(z_{\alpha} ; \rho\right)} \frac{d z_{\alpha}}{z_{\alpha}},
\end{aligned}
$$

where $z_{\alpha}$ is integrated over $\gamma_{\alpha}$ and

$$
\widetilde{\Pi}(z ; \rho)=\lim _{t \rightarrow 0} \Pi(z ; \rho)=\exp \left(\sum_{k=1}^{\infty} \frac{z^{k} p_{k}(\rho)}{k\left(1-q^{k}\right)}\right) .
$$

Remark. For the choice of $\rho$ such that $\Pi(z, \rho)=\exp (\tau z)$ for a parameter $\tau>0$, and $a_{1}=\cdots=a_{N}=1$, the formula (4.9) was guessed at and checked in [BCS]. The formulas of [BC2] are also related to some particular choices of $\rho$ in (4.9).

4.2. Comparison with formal setting. Let us compare the statements of Theorems 4.5 and 4.6 with formal statements of Section 3.

The statement of Theorem 4.6 can be obtained from Theorem 3.10 by first specializing algebras $\Lambda_{A^{i}}, \Lambda_{B^{i}}$ (see Definition 4.1), then changing the variables $z=1 / w$ and further suitably deforming the contours of integration. Note that the contours in Theorem 4.6 do not enclose 0, while in Theorem 3.10 they do. Thus, we would pick certain residues while deforming the contours, and these residues are responsible for the change in the observable. In fact, for $m=1$ we performed a similar deformation in the proof of Proposition 3.8 .

A formal version of Theorem 4.5 is more delicate. The difficulty lies in the fact that our observable $e_{r}\left(q^{\lambda_{1}} t^{k-1}, \ldots, q^{\lambda_{k}}\right)$ does not have a straightforward limit as $k \rightarrow \infty$. In the appendix (Section 6) we give a formal version of Theorem 4.5 for the case $r_{i}=1$ for all $i$. Finding such a formal version for general $r_{i}$ involves finding a suitable form of stable Macdonald operators and we do not pursue this here.

4.3. Fredholm determinants. The aim of this subsection is to present two observables of the Macdonald measure whose expectations can be written as Fredholm determinants. For the case $q=t$ (corresponding to Schur polynomials) the emergence of Fredholm determinants is well-understood, due to the identification of the Schur measure with a determinantal point process (see O1] and also BG] for a 
recent review). No such structure predicting the appearance of Fredholm determinants is known for general parameters $(q, t)$. The $t=0$ and general $q$ degeneration of the first Fredholm determinant we present in Theorem 4.8 was discovered in [BC, Corollary 3.2.10 and Theorem 3.2.11] by utilizing the first Macdonald difference operator and its powers. Our present result uses a different operator diagonalized by the Macdonald polynomials (that however degenerates to a generating series of powers of the first Macdonald operator at $t=0)$.

We will write Fredholm determinant formulas for the (ascending) Macdonald measure $\mathbb{M M}_{N,\left\{a_{i}\right\} ; \rho}^{\mathbf{a}}$. We do not approach the question of generalizing the formulas below to the formal settings of Section 3 (again this is known to be possible for the $q=t$ case).

Theorem 4.8. Fix $N$ non-zero complex numbers $a_{1}, \ldots, a_{N}$ and a specialization $\rho$ such that for some $0<R<1$ we have

$$
\left|p_{k}(\rho)\right|<R^{k}, k=1,2, \ldots, \quad\left|a_{i}\right| R<1, i=1, \ldots, N .
$$

Define the function

$$
\mathrm{G}_{N,\left\{a_{i}\right\}, \rho}(w)=\frac{1}{\Pi(w ; \rho)} \prod_{j=1}^{N} \frac{\left(t w / a_{j} ; q\right)_{\infty}}{\left(w / a_{j} ; q\right)_{\infty}} .
$$

Further, let $u$ be a formal variable and set

$$
K_{u, N,\left\{a_{i}\right\}, \rho}\left(w, w^{\prime}\right)=\sum_{v=1}^{\infty} \frac{u^{v}}{q^{v} w-w^{\prime}} \frac{\mathrm{G}_{N,\left\{a_{i}\right\}, \rho}(w)}{\mathrm{G}_{N,\left\{a_{i}\right\}, \rho}\left(q^{v} w\right)} .
$$

Suppose that there exists a positively oriented contour $\gamma$ such that:

(I) $\gamma$ lies inside $\mathbb{D}_{R^{-1}}=\left\{z \in \mathbb{C}|| z \mid<R^{-1}\right\}$.

(II) $\gamma$ encloses all points $a_{i}, i=1, \ldots, N$, but not $q^{s} t a_{i}, s=1,2, \ldots, i=$ $1, \ldots, N$.

(III) the contour $q^{k} \gamma$ is outside $\gamma$ for $k=1,2, \ldots$

(Note that when $a_{1}=\cdots=a_{N}$, a small circle around $a_{1}$ satisfies all the above assumptions.)

Then the following equality holds as an identity of power series in u:

$$
\sum_{\lambda \in \mathbb{Y}} \prod_{i=1}^{N} \frac{\left(q^{\lambda_{i}} t^{N-i+1} u ; q\right)_{\infty}}{\left(q^{\lambda_{i}} t^{N-i} u ; q\right)_{\infty}} \mathbb{M M}_{N,\left\{a_{i}\right\}, \rho}^{\mathbf{a}}(\lambda)=\operatorname{det}\left(I+K_{u, N,\left\{a_{i}\right\}, \rho}\right)_{L^{2}(\gamma)} .
$$

Remark 1. In the theorem we define the Fredholm determinant

$$
\operatorname{det}\left(I+K_{u, N,\left\{a_{i}\right\}, \rho}\right)_{L^{2}(\gamma)}
$$

through its Fredholm series expansion

$$
\begin{aligned}
\operatorname{det}(I & \left.+K_{u, N,\left\{a_{i}\right\}, \rho}\right)_{L^{2}(\gamma)} \\
& =1+\sum_{k=1}^{\infty} \frac{1}{k !} \int_{\gamma} d w_{1} \cdots \int_{\gamma} d w_{k} \operatorname{det}\left[K_{u, N,\left\{a_{i}\right\}, \rho}\left(w_{i}, w_{j}\right)\right]_{i, j=1}^{k} .
\end{aligned}
$$

Our definitions imply that the $k^{t h}$ term in the sum in (4.10) is a power series in $u$ starting from $u^{k}$. Therefore, (4.10) is a well-defined power series in $u$. In fact, this power series is easily seen in the proof to be a degree $N$ polynomial in $u$. 
Remark 2. When the above theorem is seen as an analytic identity after specifying some value of $u$ for which all the series absolutely converge, then the kernel $K_{u, N,\left\{a_{i}\right\}, \rho}$ can be represented in the form

$$
K_{u, N,\left\{a_{i}\right\}, \rho}\left(w, w^{\prime}\right)=\int_{C_{1,2, \ldots}} \Gamma(-s) \Gamma(1+s) \frac{(-u)^{s}}{q^{s} w-w^{\prime}} \frac{\mathrm{G}_{N,\left\{a_{i}\right\}, \rho}(w)}{\mathrm{G}_{N,\left\{a_{i}\right\}, \rho}\left(q^{s} w\right)}
$$

where $C_{1,2, \ldots}$ is a negatively oriented contour which encloses the positive integer poles of $\Gamma(-s) \Gamma(1+s)$ and no other poles of the integrand. This representation is useful when performing asymptotics (cf. [BC, Section 3.2.3], $\mathrm{BCF}$, Sections 5 and $6])$.

Remark 3. When $t=0$, [BC, Theorem 3.2.16] provides a second Fredholm determinant formula for the same expectation which differs from the result of taking $t=0$ in the above theorem (cf. BC, Corollary 3.2.10 and Theorem 3.2.11]). It might be possible to write an analog to this second type of Fredholm determinant at the general $(q, t)$ level, though we do not pursue that here as it is so far unclear if it has applications.

We now present the proof of Theorem 4.8, At the end of the section we state one other Fredholm determinant result in the form of Theorem 4.13

Proof of Theorem 4.8. The following operator and eigenfunction relation in Proposition 4.10 come from [FHHSY, Proposition 3.24].

Definition 4.9. For any $N \geq 1$ and any $\nu \in\left(\mathbb{Z}_{\geq 0}\right)^{N}$ define a difference operator $\mathfrak{N}_{N, \nu}^{u}$ which acts on the space of analytic functions in $x_{1}, \ldots, x_{N}$ as

$$
\mathfrak{N}_{N, \nu}^{u}=u^{|\nu|} \prod_{1 \leq i<j \leq N} \frac{q^{\nu_{j}} x_{j}-q^{\nu_{i}} x_{i}}{x_{j}-x_{i}} \prod_{1 \leq i, j \leq N} \frac{\left(t x_{i} / x_{j} ; q\right)_{\nu_{i}}}{\left(q x_{i} / x_{j} ; q\right)_{\nu_{i}}} \prod_{i=1}^{N}\left(T_{q, i}\right)^{\nu_{i}} .
$$

Define the Noumi q-integral operator $\mathfrak{N}_{N}^{u}$ which acts on the space of analytic functions in $x_{1}, \ldots, x_{N}$ as

$$
\mathfrak{N}_{N}^{u}=\sum_{\nu \in\left(\mathbb{Z}_{\geq 0}\right)^{N}} \mathfrak{N}_{N, \nu}^{u}
$$

The Macdonald polynomials diagonalize the Noumi $q$-integral operator with explicit eigenvalues.

Proposition 4.10 (Noumi). For any $N \geq 1$, formal parameter $u$, and $\lambda \in \mathbb{Y}$ such that $\ell(\lambda) \leq N$, the following identity of power series in $u$ holds:

$$
\mathfrak{N}_{N}^{u} P_{\lambda}\left(x_{1}, \ldots, x_{n}\right)=\prod_{i=1}^{N} \frac{\left(q^{\lambda_{i}} t^{N+1-i} u ; q\right)_{\infty}}{\left(q^{\lambda_{i}} t^{N-i} u ; q\right)_{\infty}} P_{\lambda}\left(x_{1}, \ldots, x_{N}\right) .
$$

This proposition is proved in Section 5. It first appeared in the literature as [FHHSY, Proposition 3.24] and therein is attributed to a personal communication from M. Noumi; its proof will appear in [NS]. Since at the time of publication this proof had not yet been posted, we also present E. Rains' proof of this result as an appendix in Section 5 . 
Remark. For $r \geq 0$, the operator

$$
\mathfrak{N}_{N}^{[r]} \sum_{\substack{\nu \in\left(\mathbb{Z}_{\geq 0}\right)^{N} \\|\nu|=r}} \mathfrak{N}_{N, \nu}^{u}
$$

is also diagonalized by the $P_{\lambda}$ with eigenvalues $g_{r}\left(q^{\lambda_{1}} t^{N-1}, q^{\lambda_{2}} t^{N-2}, \ldots, q^{\lambda_{N}} t^{0}\right)$. Here $g_{r}$ is the $(q, t)$-version of the complete homogeneous symmetric polynomial (i.e., $\left.g_{r}=Q_{(r)}\right)$. Clearly $\mathfrak{N}_{N}^{u}=\sum_{r=0}^{\infty} u^{r} \mathfrak{N}_{N}^{[r]}$.

Proposition 4.10 implies that

$$
\sum_{\lambda \in \mathbb{Y}} \prod_{i=1}^{N} \frac{\left(q^{\lambda_{i}} t^{N+1-i} u ; q\right)_{\infty}}{\left(q^{\lambda_{i}} t^{N-i} u ; q\right)_{\infty}} \mathbb{M M}_{N,\left\{a_{i}\right\} ; \rho}^{\mathbf{a}}(\lambda)=\left.\frac{\mathfrak{N}_{N}^{u} \Pi\left(x_{1}, \ldots, x_{N} ; \rho\right)}{\Pi\left(x_{1}, \ldots, x_{N} ; \rho\right)}\right|_{x_{1}=a_{1}, \ldots, x_{N}=a_{N}}
$$

the argument here being parallel to that of Proposition 4.4 see also [BC, Section 2.2.3] for a general discussion. The only thing to check here is that the series giving the coefficient of $u^{r}$ in the left side of (4.14) is absolutely convergent. This series is (cf. [M, Chapter VI, (2.8)])

$$
\sum_{\lambda \in \mathbb{Y}} g_{r}\left(q^{\lambda_{1}} t^{N-1}, q^{\lambda_{2}} t^{N-2}, \ldots, q^{\lambda_{N}} t^{0}\right) \mathbb{M}_{N,\left\{a_{i}\right\} ; \rho}^{\mathbf{a}}(\lambda) .
$$

The combinatorial formula for Macdonald polynomials $g_{r}$ (see M, Chapter VI, Section 7]) and inequalities $0<q, t<1$ imply that

$$
0 \leq g_{r}\left(q^{\lambda_{1}} t^{N-1}, q^{\lambda_{2}} t^{N-2}, \ldots, q^{\lambda_{N}} t^{0}\right) \leq g_{r}(\underbrace{1, \ldots, 1}_{N}) .
$$

Thus, 4.15) is absolutely convergent as in Definition 4.1.

Recall (cf. Section 3.1) that $\Pi\left(x_{1}, \ldots, x_{N} ; \rho\right)=\Pi\left(x_{1} ; \rho\right) \cdots \Pi\left(x_{N} ; \rho\right)$. For such functions, it is possible to encode the application of the Noumi operator in terms of a Fredholm determinant. Theorem 4.8 immediately follows from equation (4.14) along with the application of the following proposition.

Proposition 4.11. The following holds as an identity of power series in $u$ :

$$
\left.\frac{\mathfrak{N}_{N}^{u} \Pi\left(x_{1}, \ldots, x_{N} ; \rho\right)}{\Pi\left(x_{1}, \ldots, x_{N} ; \rho\right)}\right|_{x_{1}=a_{1}, \ldots, x_{N}=a_{N}}=\operatorname{det}\left(I+K_{u, N,\left\{a_{i}\right\}, \rho}\right)_{L^{2}(\gamma)} .
$$

Proof. We proceed in three steps. In step 1 we show how simple residue considerations imply that the Fredholm expansion for $\operatorname{det}\left(I+K_{u, N,\left\{a_{i}\right\}, \rho}\right)_{L^{2}(\gamma)}$ terminates after $N$ terms. In step 2 we present a lemma which relates the $k^{\text {th }}$ term in this expansion to the application of the operators $\mathfrak{N}_{N, \nu}^{u}$ with the number of non-zero parts of $\nu$ equal to $k$. In step 3 we conclude the proof by combining the two previous steps.

Step 1. Recall the definition of $\operatorname{det}\left(I+K_{u, N,\left\{a_{i}\right\}, \rho}\right)_{L^{2}(\gamma)}$ via the Fredholm series expansion. We can rewrite

$$
K_{u, N,\left\{a_{i}\right\}, \rho}\left(w, w^{\prime}\right)=\prod_{r=1}^{N} \frac{1}{w-a_{r}} \tilde{K}_{u, N,\left\{a_{i}\right\}, \rho}\left(w, w^{\prime}\right)
$$

where $\tilde{K}_{u, N,\left\{a_{i}\right\}, \rho}\left(w, w^{\prime}\right)$ is now analytic inside the contour $\gamma$ in both variables. This means that we can evaluate all of the $w_{i}$ integrations in (4.10) via the residue 
theorem. Each variable $w_{i}$ can pick a residue at any of $\left\{a_{1}, \ldots, a_{N}\right\}$. This leads to the expansion

$$
\begin{gathered}
\int_{\gamma} d w_{1} \cdots \int_{\gamma} d w_{k} \operatorname{det}\left[K_{u, N,\left\{a_{i}\right\}, \rho}\left(w_{i}, w_{j}\right)\right]_{i, j=1}^{k} \\
=\sum_{p} \operatorname{det}\left[\prod_{\substack{r=1 \\
r \neq p(i)}}^{N} \frac{1}{a_{p(i)}-a_{r}} \tilde{K}_{u}\left(a_{p(i)}, a_{p(j)}\right)\right]_{i, j=1}^{k}
\end{gathered}
$$

where the summation is over all assignments $p:\{1, \ldots k\} \rightarrow\{1, \ldots, N\}$. If $k>N$, then there must exist some $i \neq i^{\prime}$ such that $p(i)=p\left(i^{\prime}\right)$. Consequently, row $i$ and row $i^{\prime}$ of the above matrix coincide; hence the determinant is zero. Thus

$$
\begin{aligned}
\operatorname{det}\left(I+K_{u, N,\left\{a_{i}\right\}, \rho}\right)_{L^{2}(\gamma)} & \\
& =1+\sum_{k=1}^{N} \frac{1}{k !} \int_{\gamma} d w_{1} \cdots \int_{\gamma} d w_{k} \operatorname{det}\left[K_{u, N,\left\{a_{i}\right\}, \rho}\left(w_{i}, w_{j}\right)\right]_{i, j=1}^{k} .
\end{aligned}
$$

Step 2. We now show how the $k^{\text {th }}$ term in equation (4.16) arises from a combination of the $\mathfrak{N}_{N, \nu}^{u}$ with the number of non-zero parts of $\nu$ equal to $k$ (and these non-zero parts summed over the natural numbers).

Lemma 4.12. Fix $N \geq 0, k \in\{0,1, \ldots, N\}$ and assume $\nu \in\left(\mathbb{Z}_{\geq 0}\right)^{N}$ is such that $\nu_{1}, \ldots, \nu_{k} \geq 1$ and $\nu_{k+1}, \ldots, \nu_{N}=0$. Then, for all $a_{1}, \ldots, a_{N}$ and $u$,

$$
\begin{aligned}
& \left.\frac{1}{(N-k) !} \sum_{\sigma \in S_{N}} \frac{\mathfrak{N}_{N, \sigma(\nu)}^{u} \Pi\left(x_{1}, \ldots, x_{N} ; \rho\right)}{\Pi\left(x_{1}, \ldots, x_{N} ; \rho\right)}\right|_{x_{1}=a_{1}, \ldots, x_{N}=a_{N}} \\
& \quad=\frac{1}{(2 \pi \mathbf{i})^{k}} \int_{\gamma} d w_{1} \cdots \int_{\gamma} d w_{k} \operatorname{det}\left[K_{u, N,\left\{a_{i}\right\}, \rho}^{\prime}\left(\nu_{i}, w_{i}, w_{j}\right)\right]_{i, j=1}^{k},
\end{aligned}
$$

where

$$
K_{u, N,\left\{a_{i}\right\}, \rho}^{\prime}\left(v, w, w^{\prime}\right)=\frac{u^{v}}{q^{v} w-w^{\prime}} \frac{\mathrm{G}_{N,\left\{a_{i}\right\}, \rho}(w)}{\mathrm{G}_{N,\left\{a_{i}\right\}, \rho}\left(q^{v} w\right)},
$$

and $S_{n}$ is the symmetric group of rank $N$ which acts on $\nu=\left(\nu_{1}, \ldots, \nu_{k}\right)$ by permuting its coordinates.

Proof. We evaluate the right-hand side of equation (4.17) via residues in order to prove the theorem. Observe that the only term in $K_{u, N,\left\{a_{i}\right\}, \rho}^{\prime}$ involving both $w$ and $w^{\prime}$ is $\left(q^{v} w-w^{\prime}\right)^{-1}$. The Cauchy determinant identity (3.15) may be applied to this term, and a small calculation and reordering of terms leads to

$$
\begin{aligned}
\operatorname{RHS}(4.17) & =\frac{1}{(2 \pi \mathbf{i})^{k}} \int_{\gamma} d w_{1} \cdots \int_{\gamma} d w_{k} \prod_{i=1}^{k} \prod_{j=1}^{N} \frac{-1}{w_{i}-a_{j}} \\
& \times \prod_{\substack{i, j=1 \\
i \neq j}}^{k}\left(w_{i}-w_{j}\right) \prod_{i, j=1}^{k} \frac{-1}{w_{j}-q^{\nu_{i}} w_{i}} \prod_{i=1}^{k} \prod_{j=1}^{N}\left(a_{j}-q^{\nu_{i}} w_{i}\right) \\
& \times u^{|\nu|} \prod_{1 \leq i<j \leq k} \frac{q^{\nu_{i}} w_{i}-q^{\nu_{j}} w_{j}}{w_{i}-w_{j}} \prod_{i=1}^{k} \prod_{j=1}^{N} \frac{\left(t w_{i} / a_{j} ; q\right)_{\infty}}{\left(q w_{i} / a_{j} ; q\right)_{\infty}} \prod_{i=1}^{k} \frac{\Pi\left(q^{\nu_{i}} w_{i} ; \rho\right)}{\Pi\left(w_{i} ; \rho\right)} .
\end{aligned}
$$


Inspection of the above formula reveals that it is only the first product which has poles inside the $\gamma$ contour. The residue theorem implies that we can evaluate the above integral by computing the sum of the residues at $w_{i}=a_{p(i)}, 1 \leq i \leq k$, summed over every choice of assignment $p:\{1, \ldots, k\} \rightarrow\{1, \ldots, N\}$. Further inspection reveals that due to factors $\left(w_{i}-w_{j}\right)$, if $p(i)=p(j)$ for some $i \neq j$, then the residue is zero. Hence we are left with the sum over all assignments for which $p(i) \neq$ $p(j)$ when $i \neq j$. As a convention, define $p(1+k), \ldots, p(N)$ to be the (ordered) remaining elements of $\{1, \ldots, N\}$ which are not equal to $p(1), \ldots, p(k)$. Denote as $P$ the set of all such defined assignment (or permutations) from $\{1, \ldots, N\} \rightarrow$ $\{1, \ldots, N\}$. Thus (after noticing that various factors of -1 multiply to 1 )

$$
\begin{aligned}
\text { RHS (4.17) } & =\sum_{p \in P} \prod_{i=1}^{k} \prod_{j=k+1}^{N} \frac{q^{\nu_{i}} a_{p(i)}-a_{p(j)}}{a_{p(i)}-a_{p(j)}} \\
& \times u^{|\nu|} \prod_{1 \leq i<j \leq k} \frac{q^{\nu_{i}} x_{p(i)}-q^{\nu_{j}} a_{p(j)}}{a_{p(i)}-a_{p(j)}} \prod_{i=1}^{N} \prod_{j=1}^{N} \frac{\left(t a_{p(i)} / a_{p(j)} ; q\right)_{\infty}}{\left(q a_{p(i)} / a_{p(j)} ; q\right)_{\infty}} \prod_{i=1}^{k} \frac{\Pi\left(q^{\nu_{i}} a_{p(i)} ; \rho\right)}{\Pi\left(a_{p(i)} ; \rho\right)} .
\end{aligned}
$$

Recalling that $\nu_{k+1}=\ldots=\nu_{N}=0$ we can combine the above expressions as

RHS (4.17)

$$
=\sum_{p \in P} u^{|\nu|} \prod_{1 \leq i<j \leq N} \frac{q^{\nu_{i}} a_{p(i)}-q^{\nu_{j}} a_{p(j)}}{a_{p(i)}-a_{p(j)}} \prod_{i, j=1}^{N} \frac{\left(t a_{p(i)} / a_{p(j)} ; q\right)_{\infty}}{\left(q a_{p(i)} / a_{p(j)} ; q\right)_{\infty}} \prod_{i=1}^{N} \frac{\Pi\left(q^{\nu_{i}} a_{p(i)} ; \rho\right)}{\Pi\left(a_{p(i)} ; \rho\right)} .
$$

Again, due to the fact that $\nu_{k+1}=\ldots=\nu_{N}=0$, the summation over $p \in P$ can be replaced by $p \in S_{N}$, yielding

$$
\begin{aligned}
\operatorname{RHS}(4.17)=\frac{1}{(N-k) !} \sum_{p \in S_{N}} u^{|\nu|} & \prod_{1 \leq i<j \leq N} \frac{q^{\nu_{i}} a_{p(i)}-q^{\nu_{j}} a_{p(j)}}{a_{p(i)}-a_{p(j)}} \\
& \times \prod_{i, j=1}^{N} \frac{\left(t a_{p(i)} / a_{p(j)} ; q\right)_{\infty}}{\left(q a_{p(i)} / a_{p(j)} ; q\right)_{\infty}} \prod_{i=1}^{N} \frac{\Pi\left(q^{\nu_{i}} a_{p(i)} ; \rho\right)}{\Pi\left(a_{p(i)} ; \rho\right)} .
\end{aligned}
$$

The $(N-k)$ ! came from the size of $S_{N} / P$. We now recall $\sigma=p^{-1}$ and replace $a_{p(i)}$ by $a_{i}$ and $\nu_{i}$ by $\nu_{\sigma(i)}$ in the above expression. Noting that

$$
\frac{\left(T_{q, i}^{\nu_{i}} \Pi\right)\left(x_{1}, \ldots, x_{N} ; \rho\right)}{\Pi\left(x_{1}, \ldots, x_{N} ; \rho\right)}=\frac{\Pi\left(q^{\nu_{i}} x_{i} ; \rho\right)}{\Pi\left(x_{i} ; \rho\right)},
$$

we are finally led to

$$
\operatorname{RHS}(4.17)=\left.\frac{1}{(N-k) !} \sum_{\sigma \in S_{N}} \frac{\mathfrak{N}_{N, \sigma(\nu)}^{u} \Pi\left(x_{1}, \ldots, x_{N} ; \rho\right)}{\Pi\left(x_{1}, \ldots, x_{n} ; \rho\right)}\right|_{x_{1}=a_{1}, \ldots, x_{N}=a_{N}},
$$

as desired to prove the lemma.

Step 3. We now rewrite the Noumi $q$-integral operator in terms of the expressions on the left-hand side of equation (4.17). In particular, we split the summation 
defining $\mathfrak{N}_{N}^{u}$ based on the number of non-zero parts to $\nu$ :

$$
\begin{aligned}
\mathfrak{N}_{N}^{u}=\sum_{\nu \in\left(\mathbb{Z}_{\geq 0}\right)^{N}} \mathfrak{N}_{N, \nu}^{u} & =\sum_{k=0}^{N} \sum_{\substack{S \subseteq\{1, \ldots, N\} \\
|S|=k}} \sum_{\substack{\nu \in\left(\mathbb{Z}_{\geq 0}\right)^{N} \\
\nu_{i}>0 \text { for } i \in S \nu_{i}=0 \text { for } i \notin S}} \mathfrak{N}_{N, \nu}^{u} \\
& =\sum_{k=0}^{N} \sum_{\nu_{1}, \ldots \nu_{k}=1}^{\infty} \frac{N !}{(N-k) ! k !} \frac{1}{N !} \sum_{\sigma \in S_{N}} \mathfrak{N}_{N, \sigma(\nu)}^{u},
\end{aligned}
$$

where in the second line $\nu=\left(\nu_{1}, \ldots, \nu_{k}, 0, \ldots 0\right)$. Note that the $N$ choose $k$ factor came from the number of ways of choosing the subset $S$, and the reciprocal of $N$ factorial came from the symmetrization of $\nu$.

Using the above calculation and Lemma 4.12 we find that

$$
\begin{aligned}
& \left.\frac{\mathfrak{N}_{N}^{u} \Pi\left(x_{1}, \ldots, x_{N} ; \rho\right)}{\Pi\left(x_{1}, \ldots, x_{n} ; \rho\right)}\right|_{x_{1}=a_{1}, \ldots, x_{N}=a_{N}} \\
& =\sum_{k=0}^{N} \frac{1}{k !} \sum_{\nu_{1}, \ldots \nu_{k}=1}^{\infty} \frac{1}{(2 \pi \mathbf{i})^{k}} \int_{\gamma} d w_{1} \cdots \int_{\gamma} d w_{k} \operatorname{det}\left[K_{u, N,\left\{a_{i}\right\}, \rho}^{\prime}\left(\nu_{i}, w_{i}, w_{j}\right)\right]_{i, j=1}^{k} \\
& =\sum_{k=0}^{N} \frac{1}{k !} \frac{1}{(2 \pi \mathbf{i})^{k}} \int_{\gamma} d w_{1} \cdots \int_{\gamma} d w_{k} \operatorname{det}\left[K_{u, N,\left\{a_{i}\right\}, \rho}\left(w_{i}, w_{j}\right)\right]_{i, j=1}^{k},
\end{aligned}
$$

where in the third line the summation over the $\nu_{i}$ was absorbed into the determinant (resulting in the $K_{u, N,\left\{a_{i}\right\}, \rho}$ kernel). Finally, by virtue of equation (4.16) from step 1 , we conclude the proof of the proposition.

As explained before the statement of Proposition 4.11 this also completes the proof of Theorem 4.8 .

We present a second general $(q, t)$ Fredholm determinant which relies upon the Macdonald difference operators $\mathfrak{M}_{N}^{r}$ (see Section 4.1) and their elementary symmetric function eigenvalues (see equation (4.2)).

Letting $u$ be a formal parameter, we may define

$$
\mathfrak{M}_{N}(u)=\sum_{r=0}^{N}(-u)^{r} \mathfrak{M}_{N}^{r} .
$$

Then, for $\lambda \in \mathbb{Y}$ with $\ell(\lambda) \leq N$, we have

$$
\mathfrak{M}_{N}(u) P_{\lambda}\left(x_{1}, \ldots, x_{N}\right)=\prod_{i=1}^{N}\left(1-u q^{\lambda_{i}} t^{N-i}\right) .
$$

This follows from equation (4.2) since the right-hand side above is the generating function for the elementary symmetric polynomials; cf. [M, Chapter I, Section 2].

Theorem 4.13. Fix $N$ non-zero complex numbers $a_{1}, \ldots, a_{N}$ and a specialization $\rho$ such that for some $0<R<1$ we have

$$
\left|p_{k}(\rho)\right|<R^{k}, k=1,2, \ldots, \quad\left|a_{i}\right| R<1, i=1, \ldots, N .
$$


Let $C_{a}$ be a contour which lies inside a circle of radius $R^{-1}$ and which encloses all $a_{1}, \ldots, a_{N}$ but not $t a_{1}, \ldots, t a_{N}$. Set

$$
J_{N,\left\{a_{i}\right\}, \rho}\left(w, w^{\prime}\right)=\frac{1}{t w^{\prime}-w} \prod_{m=1}^{N} \frac{t w-a_{m}}{w-a_{m}} \frac{\Pi(q w ; \rho)}{\Pi(w ; \rho)} .
$$

Then the following equality holds as an identity of power series in u:

$$
\sum_{\lambda \in \mathbb{Y}} \prod_{i=1}^{N}\left(1-u q^{\lambda_{i}} t^{N-i}\right) \mathbb{M M}_{N,\left\{a_{i}\right\}, \rho}^{\mathbf{a}}(\lambda)=\operatorname{det}\left(I-u J_{N,\left\{a_{i}\right\}, \rho}\right)_{L^{2}\left(C_{a}\right)}
$$

Remark 1. The above Fredholm determinant is related to the generating function of the elementary symmetric polynomials $e_{r}$, whereas the Fredholm determinant presented in Theorem 4.8 is related (see Proposition 4.10) to the $(q, t)$-analog of the complete homogeneous symmetric polynomials $g_{r}$. There is an endomorphism $\omega_{q, t}$ on $\Lambda$ which maps $\omega_{q, t} g_{r}(X ; q, t)=e_{r}(X)$. At this point, it is not clear how this endomorphism is related to the two Fredholm determinant formulas we have presented.

Remark 2. In equation (3.3) of [W] (for $\rho$ a finite length specialization into a set of complex numbers $\left.y_{1}, y_{2}, \ldots\right)$ an alternative expression (written as $F(u ; x, y ; t)$ ) is given for the above Fredholm determinant. This function is then related to the Izergin-Korepin determinant.

Remark 3. It is possible to state a formal version of the above theorem immediately from Proposition 3.8 .

Proof of Theorem 4.13. Observe that by virtue of the eigenrelation (4.18) satisfied by $\mathfrak{M}_{N}(u)$,

$$
\left.\frac{\mathfrak{M}_{N}(u) \Pi\left(x_{1}, \ldots, x_{N} ; \rho\right)}{\Pi\left(x_{1}, \ldots, x_{N} ; \rho\right)}\right|_{x_{1}=a_{1}, \ldots, x_{N}=a_{N}}=\sum_{\lambda \in \mathbb{Y}} \prod_{i=1}^{N}\left(1-u q^{\lambda_{i}} t^{N-i}\right) \mathbb{M M}_{N,\left\{a_{i}\right\}, \rho}^{\mathbf{a}}(\lambda)
$$

A special case of Theorem 4.5 (also found in [BC, Proposition 2.2.10]) states that

$$
\begin{aligned}
& \left.\frac{\mathfrak{M}_{N}^{r} \Pi\left(x_{1}, \ldots, x_{N} ; \rho\right)}{\Pi\left(x_{1}, \ldots, x_{N} ; \rho\right)}\right|_{x_{1}=a_{1}, \ldots, x_{N}=a_{N}} \\
& \quad=\frac{1}{r !} \frac{1}{(2 \pi \mathbf{i})^{r}} \oint_{C_{a}} d w_{1} \cdots \oint_{C_{a}} d w_{r} \operatorname{det}\left[J_{N,\left\{a_{i}\right\}, \rho}\left(w_{k}, w_{\ell}\right)\right]_{k, \ell=1}^{r}
\end{aligned}
$$

Multiplying each term by $(-u)^{r}$ and summing over $r \in\{0,1, \ldots, N\}$ we recover the first $N+1$ terms in the Fredholm series expansion of $\operatorname{det}\left(I-u J_{N,\left\{a_{i}\right\}, \rho}\right)_{L^{2}\left(C_{a}\right)}$. It is easy to see that all further terms in the expansion vanish (this is somewhat similar to step 1 in the proof of Theorem 4.8), hence the desired result. 


\section{Appendix: E. Rains' proof of Proposition 4.10}

The following appendix, due to Eric Rains, provides a derivation of Proposition 4.10 from an elliptic integral operator. Let us fix some notation:

$$
\begin{gathered}
\theta_{q}(x):=\prod_{k \geq 0}\left(1-q^{k} x\right)\left(1-q^{k+1} / x\right), \quad \Gamma_{p, q}(x):=\prod_{j, k \geq 0} \frac{1-p^{j+1} q^{k+1} / x}{1-p^{j} q^{k} x} \\
\tilde{\Gamma}_{q}(x):=\frac{1}{(x ; q)_{\infty}} .
\end{gathered}
$$

Note that $\tilde{\Gamma}_{q}(x)$ is slightly different than the usual definition of the $q$-deformed Gamma function, hence the tilde. When multiple arguments come into these functions, it means that one multiplies the single variable evaluation over all variables. For example,

$$
\Gamma_{p, q}\left(y_{i}^{ \pm} y_{j}^{ \pm}\right):=\Gamma_{p, q}\left(y_{i} y_{j}\right) \Gamma_{p, q}\left(y_{i} y_{j}^{-1}\right) \Gamma_{p, q}\left(y_{i}^{-1} y_{j}\right) \Gamma_{p, q}\left(y_{i}^{-1} y_{j}^{-1}\right)
$$

or $(a, b ; q)_{\infty}=(a ; q)_{\infty}(b ; q)_{\infty}$. In what follows, a pair of partitions is denoted by a bold lambda $\boldsymbol{\lambda}$, whereas a single partition is just $\lambda$.

The elliptic interpolation functions $\mathcal{R}_{\boldsymbol{\lambda}}^{*(n)}\left(y_{1}, \ldots, y_{n} ; u_{0}, u_{1} ; t ; p, q\right)$ are defined in equation (8.45) of [R1]. They satisfy the following integral operator identity, which is itself a special case of that given in equation (8.12) of [R1]:

$$
\begin{array}{r}
\frac{\mathcal{R}_{\boldsymbol{\lambda}}^{*(n)}\left(y_{1}, \ldots, y_{n} ; u_{0}, u_{1} ; t ; p, q\right)}{\mathcal{R}_{\boldsymbol{\lambda}}^{*(n)}\left(\ldots, t^{n-i} u_{2}, \ldots ; u_{0}, u_{1} ; t ; p, q\right)}=\prod_{0 \leq r<s \leq 3} \prod_{i=1}^{n} \Gamma_{p, q}\left(t^{n-i} u_{r} u_{s}\right) \frac{\left((p ; p)_{\infty}(q ; q)_{\infty}\right)^{n}}{\left(2 \Gamma_{p, q}(t)\right)^{n} n !} \\
\times \int_{C^{n}} \frac{\mathcal{R}_{\lambda}^{*(n)}\left(x_{1}, \ldots, x_{n} ; t^{-1 / 2} u_{0}, t^{-1 / 2} u_{1} ; t ; p, q\right)}{\mathcal{R}_{\boldsymbol{\lambda}}^{*(n)}\left(\ldots, t^{n-i-1 / 2} u_{2}, \ldots ; t^{-1 / 2} u_{0}, t^{-1 / 2} u_{1} ; t ; p, q\right)} \\
\times \frac{\prod_{i, j=1}^{n} \Gamma_{p, q}\left(t^{1 / 2} x_{i}^{ \pm 1} y_{j}^{ \pm 1}\right)}{\prod_{1 \leq i<j \leq n} \Gamma_{p, q}\left(t y_{i}^{ \pm 1} y_{j}^{ \pm 1}, x_{i}^{ \pm 1} x_{j}^{ \pm 1}\right)} \\
\times \prod_{i=1}^{n} \frac{\prod_{r=0}^{3} \Gamma_{p, q}\left(t^{-1 / 2} u_{r} x_{i}^{ \pm 1}\right)}{\Gamma_{p, q}\left(x_{i}^{ \pm 2}\right) \prod_{r=0}^{3} \Gamma_{p, q}\left(u_{r} y_{i}^{ \pm 1}\right)} \frac{d x_{i}}{2 \pi \mathbf{i} x_{i}}
\end{array}
$$

which is valid under the assumption $t^{n-2} u_{0} u_{1} u_{2} u_{3}=p q$. Here the notation ". ...t $t^{n-i} u_{2} \ldots$ " means the set of variables $t^{n-i} u_{2}, i=1, \ldots, n$, and similarly for "..., $t^{n-i-1 / 2} u_{2}, \ldots$ ". The contours $C$ are constrained so that every (infinite) collection of poles which converge to 0 lies inside the contour, and every (infinite) collection of poles converging to $\infty$ lies outside the contour.

If we reparametrize

$$
\left(u_{0}, u_{1}, u_{2}, u_{3}\right) \mapsto\left(s, p^{1 / 2} u_{1}, t u, p^{1 / 2} u_{3}\right)
$$

and take the limit $p \rightarrow 0$, the interpolation functions become the (symmetric versions of) the interpolation polynomials $\bar{P}_{\lambda}^{*(n)}$ of Okounkov [O2], and we obtain the 
identity

$$
\begin{aligned}
& \frac{\bar{P}_{\lambda}^{*(n)}\left(y_{1}, \ldots, y_{n} ; q, t, s\right)}{\bar{P}_{\lambda}^{*(n)}\left(\ldots, t^{i} u, \ldots ; q, t, s\right)}=\frac{\tilde{\Gamma}_{q}\left(t^{n} u s\right)}{\tilde{\Gamma}_{q}(u s)} \frac{(q, t ; q)_{\infty}^{n}}{2^{n} n !} \\
& \times \int_{C^{n}} \frac{\bar{P}_{\lambda}^{*(n)}\left(x_{1}, \ldots, x_{n} ; q, t, t^{-1 / 2} s\right)}{\bar{P}_{\lambda}^{*(n)}\left(\ldots, t^{i-1 / 2} u, \ldots ; q, t, t^{-1 / 2} s\right)} \\
& \times \frac{\prod_{i, j=1}^{n} \tilde{\Gamma}_{q}\left(t^{1 / 2} x_{i}^{ \pm 1} y_{j}^{ \pm 1}\right)}{\prod_{1 \leq i<j \leq n} \tilde{\Gamma}_{q}\left(t y_{i}^{ \pm 1} y_{j}^{ \pm 1}, x_{i}^{ \pm 1} x_{j}^{ \pm 1}\right)} \\
& \times \prod_{i=1}^{n} \frac{\tilde{\Gamma}_{q}\left(t^{-1 / 2} s x_{i}^{ \pm 1}, t^{1 / 2} u x_{i}^{ \pm 1}\right)}{\tilde{\Gamma}_{q}\left(s y_{i}^{ \pm 1}, t u y_{i}^{ \pm 1}, x_{i}^{ \pm 2}\right)} \frac{d x_{i}}{2 \pi \mathbf{i} x_{i}}
\end{aligned}
$$

We now want to reparametrize

$$
x_{i} \rightarrow t^{-1 / 2} s x_{i}, y_{i} \rightarrow s y_{i} u \rightarrow u / s
$$

and take the limit $s \rightarrow \infty$ so that the interpolation polynomials become shifted Macdonald polynomials. This is an apparently badly behaved limit, as it involves $q$-gamma functions with arguments tending to infinity. To fix this, we observe as in Lemma 5.2 of [R2] that the $S_{n}$-invariant function

$$
\frac{\theta_{q}\left(\prod_{r=0}^{n+1} w_{r} / \prod_{i=1}^{n} x_{i}\right) \prod_{i=1}^{n} \prod_{r=0}^{n+1} \theta_{q}\left(w_{r} x_{i}\right)}{\prod_{1 \leq i<j \leq n} \theta_{q}\left(x_{i} x_{j}\right) \prod_{0 \leq r<s \leq n+1} \theta_{q}\left(w_{r} w_{s}\right)^{-1}}
$$

becomes 1 if we sum over cosets of $S_{n}$ in the hyperoctahedral group $B C_{n}$. Thus if we multiply the integrand by $2^{n}$ times an instance of this function, the integral will be unchanged. Using the reflection identity

$$
\tilde{\Gamma}_{q}(x) \theta_{q}(x)=\tilde{\Gamma}_{q}(q / x)^{-1}
$$

we find that we can cancel the badly scaling gamma factors by taking $w_{0}=t^{-1 / 2} s$, $w_{n+1}=t^{1 / 2} u$, and $w_{i}=t^{1 / 2} y_{i}$ for $1 \leq i \leq n$. In particular, we find

$$
\begin{aligned}
& \frac{\bar{P}_{\lambda}^{*(n)}\left(y_{1}, \ldots, y_{n} ; q, t, s\right)}{\bar{P}_{\lambda}^{*(n)}\left(\ldots, t^{i} u, \ldots ; q, t, s\right)}=\tilde{\Gamma}_{q}\left(t^{n} u s, q / u s\right) \frac{(q, t ; q)_{\infty}^{n}}{n !} \\
& \times \int_{C^{n}} \frac{\bar{P}_{\lambda}^{*(n)}\left(x_{1}, \ldots, x_{n} ; q, t, t^{-1 / 2} s\right)}{\bar{P}_{\lambda}^{*(n)}\left(\ldots, t^{i-1 / 2} u, \ldots ; q, t, t^{-1 / 2} s\right)} \theta_{q}\left(u s t^{n / 2} \prod_{i=1}^{n} y_{i} / x_{i}\right) \\
& \quad \times \prod_{1 \leq i<j \leq n} \frac{\tilde{\Gamma}_{q}\left(q / t y_{i} y_{j}, q / x_{i} x_{j}\right)}{\tilde{\Gamma}_{q}\left(t y_{i} / y_{j}, t y_{j} / y_{i}, t / y_{i} y_{j}, x_{i} / x_{j}, x_{j} / x_{i}, 1 / x_{i} x_{j}\right)} \\
& \times \prod_{i, j=1}^{n} \frac{\tilde{\Gamma}_{q}\left(t^{1 / 2} x_{i} / y_{j}, t^{1 / 2} y_{j} / x_{i}, t^{1 / 2} / x_{i} y_{j}\right)}{\tilde{\Gamma}_{q}\left(q / t^{1 / 2} x_{i} y_{j}\right)} \\
& \quad \times \prod_{i=1}^{n} \frac{\tilde{\Gamma}_{q}\left(q / s y_{i}, q / t u y_{i}, t^{-1 / 2} s / x_{i}, t^{1 / 2} u / x_{i}, q / x_{i}^{2}\right)}{\tilde{\Gamma}_{q}\left(s / y_{i}, t u / y_{i}, q / t^{-1 / 2} s x_{i}, q / t^{1 / 2} u x_{i}, 1 / x_{i}^{2}\right)} \frac{d x_{i}}{2 \pi \mathbf{i} x_{i}},
\end{aligned}
$$


which after rescaling gives the limit

$$
\begin{aligned}
& \prod_{1 \leq i \leq n} \frac{\left(q^{\lambda_{i}} t^{n+1-i} u ; q\right)_{\infty}}{\left(q^{\lambda_{i}} t^{n-i} u ; q\right)_{\infty}} t^{|\lambda|} \bar{P}_{\lambda}^{*(n)}\left(y_{1}, \ldots, y_{n} ; q, t\right) \\
& \quad=\frac{(q, t ; q)_{\infty}^{n}}{n !} \int_{C^{n}} \bar{P}_{\lambda}^{*(n)}\left(x_{1}, \ldots, x_{n} ; q, t\right) \frac{\theta_{q}\left(u t^{n} \prod_{i=1}^{n} y_{i} / x_{i}\right)}{\theta_{q}(u)} \\
& \quad \times \frac{\prod_{i, j=1}^{n} \tilde{\Gamma}_{q}\left(x_{i} / y_{j}, t y_{j} / x_{i}\right)}{\prod_{1 \leq i<j \leq n} \tilde{\Gamma}_{q}\left(x_{i} / x_{j}, x_{j} / x_{i}, t y_{i} / y_{j}, t y_{j} / y_{i}\right)} \prod_{i=1}^{n} \frac{\tilde{\Gamma}_{q}\left(q / t u y_{i}, 1 / x_{i}\right)}{\tilde{\Gamma}_{q}\left(1 / y_{i}, q / u x_{i}\right)} \frac{d x_{i}}{2 \pi \mathbf{i} x_{i}},
\end{aligned}
$$

again with the contour containing all "small" poles and excluding all "large" poles.

Now if we rescale $x \rightarrow v x, y \rightarrow t^{-1} v y$ and take the limit $v \rightarrow \infty$, the shifted Macdonald polynomials become Macdonald polynomials, and we obtain the identity

$$
\begin{aligned}
& \prod_{i=1}^{n} \frac{\left(q^{\lambda_{i}} t^{n+1-i} u ; q\right)_{\infty}}{\left(q^{\lambda_{i}} t^{n-i} u ; q\right)_{\infty}} P_{\lambda}\left(y_{1}, \ldots, y_{n} ; q, t\right) \\
&=\frac{(q, t ; q)_{\infty}^{n}}{n !} \int_{C^{n}} P_{\lambda}\left(x_{1}, \ldots, x_{n} ; q, t\right) \frac{\theta_{q}\left(u \prod_{i=1}^{n} y_{i} / x_{i}\right)}{\theta_{q}(u)} \\
& \quad \times \frac{\prod_{i, j=1}^{n} \tilde{\Gamma}_{q}\left(t x_{i} / y_{j}, y_{j} / x_{i}\right)}{\prod_{1 \leq i<j \leq n} \tilde{\Gamma}_{q}\left(x_{i} / x_{j}, x_{j} / x_{i}, y_{i} / y_{j}, y_{j} / y_{i}\right)} \prod_{i=1}^{n} \frac{d x_{i}}{2 \pi \mathbf{i} x_{i}} .
\end{aligned}
$$

At this point, we observe that the "small" poles are at the points of the form $q^{k} y_{i}, k \geq 0$, and if we take a residue at one such point, the corresponding poles will not appear in the residual integrand. Moreover, if we shrink the contour by ever larger powers of $q$, the integrand converges to 0 exponentially fast. We may thus replace the contour-integral by a sum over residues. Note that this involves a choice of bijection between the $x$ variables and the $y$ variables, which can be absorbed by symmetry, eliminating the $1 / n$ ! factor. We thus obtain the claimed result of Proposition 4.10 .

$$
\begin{aligned}
& \prod_{i=1}^{n} \frac{\left(q^{\lambda_{i}} t^{n+1-i} u ; q\right)_{\infty}}{\left(q^{\lambda_{i}} t^{n-i} u ; q\right)_{\infty}} P_{\lambda}\left(y_{1}, \ldots, y_{n} ; q, t\right) \\
& \quad=\sum_{\nu \in\left(\mathbb{Z}_{\geq 0}\right)^{n}} u^{|\nu|} \prod_{1 \leq i<j \leq n} \frac{q^{\nu_{j}} y_{j}-q^{\nu_{i}} y_{i}}{y_{j}-y_{i}} \prod_{i, j=1}^{n} \frac{\left(t y_{i} / y_{j} ; q\right)_{\nu_{i}}}{\left(q y_{i} / y_{j} ; q\right)_{\nu_{i}}} P_{\lambda}\left(q^{\nu_{1}} y_{1}, \ldots, q^{\nu_{n}} y_{n} ; q, t\right) .
\end{aligned}
$$

Comparing coefficients of $u^{k}$ gives the eigenfunction relation of [NS, [FHHSY, Proposition 3.24].

\section{Appendix: On a formal version of Theorem 4.5}

The goal of this section is to obtain a formal version of Theorem 4.5 for the case $r_{i}=1$ for all $i$.

Theorem 6.1. Set

$$
\widehat{\mathcal{E}}_{1}(\lambda)=1+(1-t) \sum_{j=1}^{\infty}\left(1-q^{\lambda_{j}}\right) t^{-j}
$$


We have

$$
\begin{aligned}
& \text { (6.1) } \sum_{\lambda^{1}, \ldots, \lambda^{N}} \mathbb{M P}_{N, \mathbf{A}, \mathbf{B}}^{\mathbf{f}}\left(\lambda^{1}, \ldots, \lambda^{N}\right) \widehat{\mathcal{E}}_{1}\left(\lambda^{1}\right) \cdots \widehat{\mathcal{E}}_{1}\left(\lambda^{N}\right) \\
& =\frac{1}{(2 \pi \mathbf{i})^{N}} \oint \cdots \oint \prod_{\alpha=1}^{N} \frac{d v_{\alpha}}{v_{\alpha}} \prod_{1 \leq \alpha \leq \beta \leq N} \mathrm{H}^{-1}\left(\left(t v_{\alpha}\right)^{-1} ; B^{\beta}\right) \mathrm{H}^{-1}\left(A^{\alpha} ; v_{\beta}\right) \mathrm{W}\left(\left(t v_{\alpha}\right)^{-1} ; v_{\beta}\right),
\end{aligned}
$$

where $v_{\alpha}$ is integrated over the circle of radius $R_{\alpha}$ around the origin and $R_{\beta} /\left(t R_{\alpha}\right)<$ 1 for $\alpha<\beta$.

Remark 1. Formula (6.1) should be understood in the same sense as the statement of Theorem 3.10 .

Remark 2. Through suitable specializations and contour deformations Theorem 6.1 implies the statement of Theorem 4.5 for the case $r_{i}=1$ for all $i$. In particular, we could also obtain Corollary 4.7 by further setting $t=0$.

Sketch of the proof of Theorem 6.1. We start from [BC, Proposition 2.2.10] (that is also Theorem 4.5 with $m=1$ and $r=1$ ), which reads for $X=\left(x_{1}, \ldots, x_{N}\right)$ and $Y=\left(y_{1}, \ldots, y_{N}\right)$ :

$$
\begin{aligned}
& \sum_{\lambda \in \mathbb{Y}}\left(\sum_{j=1}^{N} q^{\lambda_{j}} t^{N-j}\right) P_{\lambda}(X) Q_{\lambda}(Y) \\
& =\Pi(X ; Y) \frac{t^{N}}{(t-1)} \frac{1}{2 \pi \mathbf{i}} \oint \prod_{j=1}^{N} \frac{1-t^{-1} z^{-1} x_{j}}{1-z^{-1} x_{j}} \prod_{j=1}^{N} \frac{1-z y_{j}}{1-t z y_{j}} \frac{d z}{z} \\
& =\Pi(X ; Y) \frac{t^{N}}{(t-1)} \frac{1}{2 \pi \mathbf{i}} \oint \mathrm{H}^{-1}\left((t z)^{-1} ; X\right) \mathrm{H}^{-1}(z ; Y) \frac{d z}{z},
\end{aligned}
$$

where $x_{i}$ and $y_{i}$ are assumed to be sufficiently small, and integration goes over the contours enclosing the poles at $x_{i}$ and no other poles of the integrand.

Note that the residue of the last integral at $z=0$ is $t^{-N}$. Therefore, using

$$
\sum_{j=1}^{N} t^{N-j}=\frac{1}{1-t}-\frac{t^{N}}{1-t}
$$

we can rewrite (assuming that $q^{\lambda_{k}}=1$ for $k>N$ )

$$
\begin{aligned}
& \sum_{\lambda \in \mathbb{Y}}\left(\frac{1}{t-1}+\sum_{j=1}^{\infty}\left(q^{\lambda_{j}}-1\right) t^{-j}\right) P_{\lambda}(X) Q_{\lambda}(Y) \\
& \quad=\frac{\Pi(X ; Y)}{2 \pi \mathbf{i}(t-1)} \oint \mathrm{H}^{-1}\left((t z)^{-1} ; X\right) \mathrm{H}^{-1}(z ; Y) \frac{d z}{z}
\end{aligned}
$$

with integration going over a circle around the origin. Now we can send the number of variables to infinity in (6.2) and obtain for two infinite sets of variables $X, Y$ :

$$
\begin{aligned}
\sum_{\lambda \in \mathbb{Y}}\left(1+(1-t) \sum_{j=1}^{\infty}\left(1-q^{\lambda_{j}}\right) t^{-j}\right) P_{\lambda}(X) Q_{\lambda}(Y) \\
\quad=\Pi(X ; Y) \frac{1}{(2 \pi \mathbf{i})} \oint \mathrm{H}^{-1}\left((t z)^{-1} ; X\right) \mathrm{H}^{-1}(z ; Y) \frac{d z}{z} .
\end{aligned}
$$


Since (6.3) has the same form as (3.8) we get an analogue of Theorem 3.10 (which is our Theorem 6.1) by repeating the same steps as in its proof.

\section{ACKNOWLEDGEMEnTs}

The authors appreciate helpful comments and a close reading by the referee. The first author was partially supported by NSF grant DMS-1056390. The second author was partially supported by the NSF through DMS-1208998, as well as by Microsoft Research through the Schramm Memorial Fellowship, and by a Clay Mathematics Institute Research Fellowship. The third author was partially supported by RFBR-CNRS grant 11-01-93105. The fourth author was supported in part by grant RFBR 13-02-00478, grant for support of scientific schools NSH3349.2012 .2 , and government contract 8606 .

\section{REFERENCES}

[A] A. Aggrawal, Correlation functions of the Schur process through Macdonald difference operators. In preparation.

[ACQ] G. Amir, I. Corwin, and J. Quastel, Probability distribution of the free energy of the continuum directed random polymer in $1+1$ dimensions, Comm. Pure Appl. Math. 64 (2011), no. 4, 466-537, DOI 10.1002/cpa.20347. MR2796514(2012b:60304)

[B] A. M. Borodin, Limit Jordan normal form of large triangular matrices over a finite field (Russian), Funktsional. Anal. i Prilozhen. 29 (1995), no. 4, 72-75, DOI 10.1007/BF01077476; English transl., Funct. Anal. Appl. 29 (1995), no. 4, 279-281 (1996). MR 1375543 (97c:11036)

[BC] A. Borodin and I. Corwin, Macdonald processes, Probab. Theory Related Fields 158 (2014), no. 1-2, 225-400, DOI 10.1007/s00440-013-0482-3. MR3152785

[BC2] A. Borodin and I. Corwin, Discrete time q-TASEPs, Int. Math Rev. Not., to appear, arXiv:1305.2972.

[BCF $] \quad$ A. Borodin, I. Corwin, and P. Ferrari, Free energy fluctuations for directed polymers in random media in $1+1$ dimension, Comm. Pure Appl. Math. 67 (2014), no. 7, 1129-1214. MR3207195

[BCFV] A. Borodin, I. Corwin, P. L. Ferrari, and B. Vető, Stationary solution of $1 d$ KPZ equation. In preparation.

[BCR] A. Borodin, I. Corwin, and D. Remenik, Log-gamma polymer free energy fluctuations via a Fredholm determinant identity, Comm. Math. Phys. 324 (2013), no. 1, 215-232, DOI 10.1007/s00220-013-1750-x. MR.3116323

[BCS] A. Borodin, I. Corwin, and T. Sasamoto, From duality to determinants for q-TASEP and ASEP, Ann. Probab. 42 (2014), no. 6, 2314-2382, DOI 10.1214/13-AOP868. MR.3265169

[BG] A. Borodin and V. Gorin, Lectures on integrable probability, arXiv:1212.3351.

[BG2] A. Borodin and V. Gorin, General $\beta$ Jacobi corners process and the Gaussian free field, arXiv:1305.3627.

[BO] A. Borodin and G. Olshanski, Z-measures on partitions and their scaling limits, European J. Combin. 26 (2005), no. 6, 795-834, DOI 10.1016/j.ejc.2004.06.003. MR2143199 (2006d:60018)

[BP] A. Borodin and L. Petrov, Nearest neighbor Markov dynamics on Macdonald processes, arXiv:1305.5501.

[COSZ] I. Corwin, N. O'Connell, T. Seppäläinen, and N. Zygouras, Tropical combinatorics and Whittaker functions, Duke Math. J. 163 (2014), no. 3, 513-563, DOI 10.1215/001270942410289. MR 3165422

[CP] I. Corwin and L. Petrov, The q-PushASEP: A new integrable model for traffic in $1+1$ dimension, arXiv:1308.3124.

[GLO] A. Gerasimov, D. Lebedev, and S. Oblezin, On q-deformed $\mathfrak{g l}_{l+1}$-Whittaker function. I, II, III, Comm. Math. Phys. 294 (2010), no. 1, 97-119, DOI 10.1007/s00220-009-0917y, MR2575477 (2011d:17023); ibid, 121-143. MR2575478 (2011d:17024); Lett. Math. Phys. 97 (2011), no. 1, 1-24. MR2802312 (2012f:17030). 
[FHHSY] B. Feigin, K. Hashizume, A. Hoshino, J. Shiraishi, and S. Yanagida, A commutative algebra on degenerate $\mathbb{C P}^{1}$ and Macdonald polynomials, J. Math. Phys. 50 (2009), no. 9, 095215, 42, DOI 10.1063/1.3192773. MR2566895 (2011c:33041)

[FR] P. J. Forrester and E. M. Rains, Interpretations of some parameter dependent generalizations of classical matrix ensembles, Probab. Theory Related Fields 131 (2005), no. 1, 1-61, DOI 10.1007/s00440-004-0375-6. MR2105043 (2006g:05222)

[F1] J. Fulman, Probabilistic measures and algorithms arising from the Macdonald symmetric functions, arXiv:math/971223.

[F2] J. Fulman, Random matrix theory over finite fields, Bull. Amer. Math. Soc. (N.S.) 39 (2002), no. 1, 51-85, DOI 10.1090/S0273-0979-01-00920-X. MR.1864086(2002i:60012)

[GKV] V. Gorin, S. Kerov, and A. Vershik, Finite traces and representations of the group of infinite matrices over a finite field, Adv. Math. 254 (2014), 331-395, DOI 10.1016/j.aim.2013.12.028. MR 3161102

[GS] V. Gorin and M. Shkolnikov, Multilevel Dyson Brownian Motions via Jack polynomials. In preparation.

[Ke1] S. Kerov, The boundary of Young lattice and random Young tableaux, Formal power series and algebraic combinatorics (New Brunswick, NJ, 1994), DIMACS Ser. Discrete Math. Theoret. Comput. Sci., vol. 24, Amer. Math. Soc., Providence, RI, 1996, pp. 133158. MR 1363510 (96i:05177)

[Ke2] S. V. Kerov, Asymptotic representation theory of the symmetric group and its applications in analysis, translated from the Russian manuscript by N. V. Tsilevich, with a foreword by A. Vershik and comments by G. Olshanski, Translations of Mathematical Monographs, vol. 219, American Mathematical Society, Providence, RI, 2003. MR.1984868 (2005b:20021)

[KOO] S. Kerov, A. Okounkov, and G. Olshanski, The boundary of the Young graph with Jack edge multiplicities, Internat. Math. Res. Notices 4 (1998), 173-199, DOI 10.1155/S1073792898000154. MR.1609628 (99f:05120)

[Ki] J. F. C. Kingman, Random partitions in population genetics, Proc. Roy. Soc. London Ser. A 361 (1978), no. 1704, 1-20. MR0526801 (58 \#26167)

[K] C. Krattenthaler, Advanced determinant calculus, The Andrews Festschrift (Maratea, 1998). Sém. Lothar. Combin. 42 (1999), Art. B42q, 67 pp. (electronic). MR1701596 (2002i:05013)

[M] I. G. Macdonald, Symmetric functions and Hall polynomials, 2nd ed., with contributions by A. Zelevinsky, Oxford Science Publications, Oxford Mathematical Monographs, The Clarendon Press, Oxford University Press, New York, 1995. MR1354144 (96h:05207)

[NS] M. Noumi and A. Sano, An infinite family of higher-order difference operators that commute with Ruijsenaars operators of type A. In preparation.

[OC] N. O'Connell, Directed polymers and the quantum Toda lattice, Ann. Probab. 40 (2012), no. 2, 437-458, DOI 10.1214/10-AOP632. MR2952082

[OCY] N. O'Connell and M. Yor, Brownian analogues of Burke's theorem, Stochastic Process. Appl. 96 (2001), no. 2, 285-304, DOI 10.1016/S0304-4149(01)00119-3. MR.1865759 (2002h:60175)

[O1] A. Okounkov, Infinite wedge and random partitions, Selecta Math. (N.S.) 7 (2001), no. 1, 57-81, DOI 10.1007/PL00001398. MR1856553(2002f:60019)

[O2] A. Okounkov, BC-type interpolation Macdonald polynomials and binomial formula for Koornwinder polynomials, Transform. Groups 3 (1998), no. 2, 181-207, DOI 10.1007/BF01236432. MR1628453 (99h:33061)

[OO] A. Okounkov and G. Olshanski, Asymptotics of Jack polynomials as the number of variables goes to infinity, Internat. Math. Res. Notices 13 (1998), 641-682, DOI 10.1155/S1073792898000403. MR.1636541(2001i:05156)

[OR] A. Okounkov and N. Reshetikhin, Correlation function of Schur process with application to local geometry of a random 3-dimensional Young diagram, J. Amer. Math. Soc. 16 (2003), no. 3, 581-603 (electronic), DOI 10.1090/S0894-0347-03-00425-9. MR.1969205 (2004b:60033)

[P] L. A. Petrov, A two-parameter family of infinite-dimensional diffusions on the Kingman simplex (Russian, with Russian summary), Funktsional. Anal. i Prilozhen. 43 (2009), no. 4, 45-66, DOI 10.1007/s10688-009-0036-8; English transl., Funct. Anal. Appl. 43 (2009), no. 4, 279-296. MR.2596654(2011c:60259) 
[R1] E. M. Rains, Transformations of elliptic hypergeometric integrals, Ann. of Math. (2) 171 (2010), no. 1, 169-243, DOI 10.4007/annals.2010.171.169. MR2630038(2011i:33046)

[R2] E. M. Rains, Limits of elliptic hypergeometric integrals, Ramanujan J. 18 (2009), no. 3, 257-306, DOI 10.1007/s11139-007-9055-3. MR2495549(2010b:33021)

[SS] T. Sasamoto and H. Spohn, One-dimensional KPZ equation: an exact solution and its universality, Phys. Rev. Lett. 104 (2010), 23.

[Se] T. Seppäläinen, Scaling for a one-dimensional directed polymer with boundary conditions, Ann. Probab. 40 (2012), no. 1, 19-73, DOI 10.1214/10-AOP617. MR2917766

[Sh] J. Shiraishi, A family of integral transformations and basic hypergeometric series, Comm. Math. Phys. 263 (2006), no. 2, 439-460, DOI 10.1007/s00220-005-1504-5. MR2207651 (2007c:33019)

[V] M. Vuletić, A generalization of MacMahon's formula, Trans. Amer. Math. Soc. 361 (2009), no. 5, 2789-2804, DOI 10.1090/S0002-9947-08-04753-3. MR2471939 (2009m:05192)

[W] S. O. Warnaar, Bisymmetric functions, Macdonald polynomials and $\mathfrak{s l}_{3}$ basic hypergeometric series, Compos. Math. 144 (2008), no. 2, 271-303, DOI 10.1112/S0010437X07003211. MR2406113(2009d:33051)

[Z] A. V. Zelevinsky, Representations of finite classical groups, A Hopf algebra approach, Lecture Notes in Mathematics, vol. 869, Springer-Verlag, Berlin-New York, 1981. MR643482 (83k:20017)

Department of Mathematics, Massachusetts Institute of Technology, Cambridge, Massachusetts 02139 - And - Institute for Information Transmission Problems of RusSian ACAdemy of Sciences, Moscow, Russia

E-mail address: borodin@math.mit.edu

Department of Mathematics, Columbia University, 2990 Broadway, New York, New York 10027 - And - Clay Mathematics Institute, 10 Memorial Boulevard, Suite 902, Providence, Rhode Island 02903 - And - Institut Henri Poincaré, 11 Rue Pierre et Marie Curie, 75005 Paris, France

E-mail address: ivan.corwin@gmail.com

Department of Mathematics, Massachusetts Institute of Technology, Cambridge, Massachusetts 02139 - And - Institute for Information Transmission Problems of RusSiAn ACADEMy of Sciences, Moscow, Russia

E-mail address: vadicgor@gmail.com

Department of Mathematics, University of California Berkeley, Berkeley, CaliforNIA 94720

E-mail address: shakirov@itep.ru 